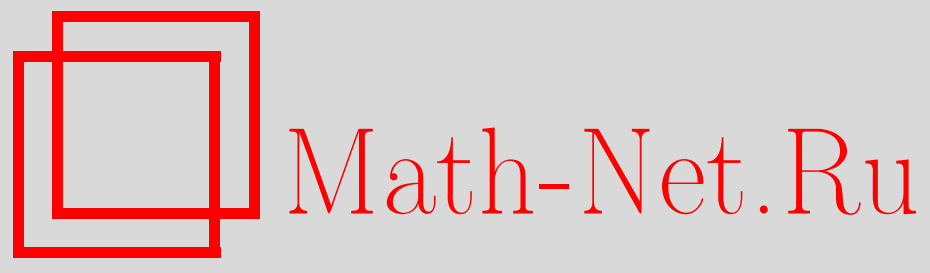

M. В. Болдин, W. Stute, O знаковых тестах в ARMA модели с возможно бесконечной дисперсией ошибок, Теория вероятн. и ее примен., 2004, том 49, выпуск 3, 436-460

DOI: https://doi.org/10.4213/tvp202

Использование Общероссийского математического портала MathNet.Ru подразумевает, что вы прочитали и согласны с пользовательским соглашением

http://www . mathnet.ru/rus/agreement

Параметры загрузки:

IP : 52.205 .19 .152

26 апреля 2023 г., 17:18:40

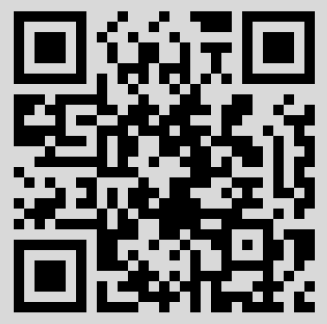




\section{О ЗНАКОВЫХ ТЕСТАХ В АRМА МОДЕЛИ С ВОЗМОЖНО БЕСКОНЕЧНОЙ ДИСПЕРСИЕЙ ОШИБОК ${ }^{1)}$}

Для ARMA модели с возможно бесконечной дисперсией строятся знаковые тесты для линейных гипотез относительно вектора неизвестных параметров. Исследуется асимптотическая мощность тестов.

Ключәвые слова и фразы: ARMA модель, бесконечная дисперсия, знаковые и ранговые тесты, асимптотическая равномерная линейность.

1. Введение. В этой работе мы рассматриваем проверку гипотез в $\operatorname{ARMA}(p, q)$ модели

$$
u_{t}=a_{1} u_{t-1}+\cdots+a_{p} u_{t-p}+\varepsilon_{t}+b_{1} \varepsilon_{t-1}+\cdots+b_{q} \varepsilon_{t-q}, \quad t \in \mathbf{Z} .
$$

Случайные величины $\left\{\varepsilon_{t}\right\}$ независимы и одинаково распределены с неизвестной функцией распределения $G, \mathbf{a}^{T}=\left(a_{1}, \ldots, a_{p}\right)$ и $\mathbf{b}^{T}=$ $\left(b_{1}, \ldots, b_{q}\right)$ - векторы неизвестных параметров, и $T$ означает транспонирование. Пусть $\mathbf{c}^{T}=\left(\mathbf{a}^{T}, \mathbf{b}^{T}\right)$. Мы рассматриваем знаковые тесты для гипотез относительно с, в том числе линейных гипотез. В отличие от традиционных постановок подобных задач мы рассматриваем ситуацию с возможно бесконечной дисперсией величин $\varepsilon_{t}$.

Обратимся сначала к стандартной ситуации. Пусть $\mathbf{E} \varepsilon_{1}=0$, $\mathbf{E} \varepsilon_{1}^{2}<\infty$. Предположим, что соответствующие (1.1) характеристические полиномы не имеют общих корней, и последовательность $\left\{u_{t}\right\}$, удовлетворяющая (1.1), каузальна и обратима, определение см. в $[4, \S 3.1]$. Пусть мы наблюдаем $u_{1}, \ldots, u_{n}$, и пусть $\left\{U_{k n}=\sum_{t=k+1}^{n} u_{t-k} u_{t} / \sum_{t=1}^{n} u_{t}^{2}\right.$, $k>0\}$ и $\left\{U_{k}\right\}$ - выборочные и истинные автокорреляции. Классические тесты для гипотез относительно с основываются на $\left\{U_{k n}\right\}$ при $q=0$ и на

* Московский государственный университет им. М.В. Ломоносова, механикоматематический факультет, кафедра теории вероятностей, Ленинские горы, 119992 Москва, Россия; e-mail: boldin_m@hotmail.com

** Mathematical Institute, Justus-Liebig-University of Giessen, Arndtstr. 2, D-35392 Giessen, Germany.

1) Работа выполнена при финансовой поддержке гранта Deutsche Forschungsgemeinschaft. 
автокорреляциях остатков при $q>0$ в предположении, что $\varepsilon_{1}$ имеет гауссовское распределение. В этом случае классические тесты оптимальны в асимптотическом локально максиминном смысле (см., например, [19], $[12, \S 4],[13])$. (Всюду в этой работе пределы берутся при $n \rightarrow \infty$ без особого упоминания.) В общем случае величины $\left\{n^{1 / 2}\left(U_{k n}-U_{k}\right)\right\}$ асимптотически нормальны. Те же тестовые статистики, что используются в гауссовском случае, имеют асимптотическое нормальное или хи-квадрат распределение, см., например, [1, гл. 5], [4, гл. 8], [19], [13], [14, §6].

Случай, когда $\mathbf{E} \varepsilon_{1}^{2}=\infty$, привлекал внимание давно. Он интересен для приложений, см., например, библиографию в [5], и любопытен для теоретического исследования. В частности, в [24] рассматривалась $\mathrm{AR}(p)$ модель с симметричной относительно нуля функцией $G$ и $\mathbf{E} \log ^{+}\left|\varepsilon_{1}\right|<\infty$. Показано, что последовательность $n^{1 / 2}\left(U_{k n}-U_{k}\right)=$ $O_{\mathbf{P}}(1)$, т.е. ограничена по вероятности. Уточнения были получены в случае, когда $G$ имеет регулярно меняющиеся индексы $\alpha$, скажем, хвосты. В [16], [15] показано, что для $\mathrm{AR}(p)$ модели при $0<\alpha<2$ (тогда $\left.\mathbf{E} \varepsilon_{1}^{2}=\infty\right)$ для любого $\delta>\alpha$ последовательность $n^{1 / \delta}\left(U_{k n}-U_{k}\right)=o_{\mathbf{P}}(1)$, т.е. сходится к нулю по вероятности. В [5] этот результат уточнен: существует медленно меняющаяся на бесконечности функция $L$, такая что $n^{1 / \alpha} L(n)\left(U_{k n}-U_{k}\right)$ сходится по распределению к частному двух независимых устойчивых случайных величин индексов $\alpha$ и $\alpha / 2$; для устойчивой функции $G$ справедливо $L(n)=(\log n)^{-1 / \alpha}$. Похожие утверждения верны и для оценок Юла-Уолкера неизвестных параметров. Родственные результаты можно найти в [2], [22], [5], [21].

Итак, если функция $G$ имеет регулярно меняющиеся хвосты и $\mathrm{E} \varepsilon_{1}^{2}=\infty$, выборочные автокорреляции сходятся даже быстрее, чем при $\mathbf{E} \varepsilon_{1}^{2}<\infty$. В такой ситуации классические тестовые статистики сходятся к нулю при гипотезах. Это влечет простое следствие. А именно, будем использовать для построения тестов гауссовскую аппроксимацию с любым фиксированным уровнем значимости тем же самым образом, как при $\mathbf{E} \varepsilon_{1}^{2}<\infty$. Тогда верные гипотезы будут приниматься с вероятностью, стремящейся к единице, и тесты будут состоятельными против фиксированных альтернатив. Разумеется, они имеют нетривиальную асимптотическую мощность только при отклонениях параметров от гипотезы на величину порядка $n^{-1 / 2}$, но не чувствительны к альтернативам, отклоняющимся от гипотезы на $o\left(n^{-1 / 2}\right)$. Тем не менее, с учетом этих замечаний классические тестовые статистики и гауссовская аппроксимация все еще могут использоваться, если $G$ имеет регулярно меняющиеся хвосты. Но что делать в более общей ситуации?

В этой работе мы строим знаковые тесты в ARMA модели (1.1) в предположении $\mathbf{E}\left|\varepsilon_{1}\right|<\infty$. Предположений о регулярности хвостов $G$ не делается. Взамен симметрии $G$ мы требуем, чтобы $\mathbf{P}\left\{\varepsilon_{1}<0\right\}=\mathbf{P}\left\{\varepsilon_{1}>\right.$ $0\}=\frac{1}{2}$, и $\mathbf{E} \varepsilon_{1}=0$. 
В разделе 2 мы начинаем с проверки гипотезы $H_{0}: \mathbf{c}=\mathbf{c}_{0}$, где $\mathbf{c}_{0}$ известный вектор, против гипотезы $H_{1}: \mathbf{c} \neq \mathbf{c}_{0}$. Чтобы построить тест, мы рассматриваем (временно, только в разделе 2) уравнение (1.1) для $t=1,2, \ldots$ с нулевыми начальными условиями. Пусть $\varepsilon_{t}\left(\mathbf{c}_{0}\right)$ будут остатки, получаемые подстановкой $\mathbf{c}_{0}$ вместо с в $(1.1)$. Тогда $\varepsilon_{t}\left(\mathbf{c}_{0}\right)=\varepsilon_{t}$ при $H_{0}$, и знаки остатков $S_{t}\left(\mathbf{c}_{0}\right)=\operatorname{sign} \varepsilon_{t}\left(\mathbf{c}_{0}\right)$ распределены свободно: $\mathbf{P}_{\mathbf{c}_{0}}\left\{S_{t}\left(\mathbf{c}_{0}\right)= \pm 1\right\}=\frac{1}{2}$. Любая тестовая статистика, основанная на векторе знаков $\mathbf{S}^{n}\left(\mathbf{c}_{0}\right)=\left(S_{1}\left(\mathbf{c}_{0}\right), \ldots, S_{n}\left(\mathbf{c}_{0}\right)\right)$ будет распределена свободно при гипотезе $H_{0}$ для фиксированных $n$. Мы конструируем тестовую статистику в два шага: сначала строим $p+q$ локально наиболее мощных для данного фиксированного $n$ (см. $[8, \S 2.4]$ для определений) статистик для $H_{0}$ при одномерных альтернативах, а потом образуем квадратическую форму от них. Наши знаковые статистики имеют (асимптотически) ту же структуру, что известные квадратические ранговые статистики и получаются заменой ранговых автокорреляций на знаковые. Оптимальные линейные и квадратические ранговые статистики для сериальной зависимости и для проверки одной ARMA модели против другой были получены в [9]-[11] для случая конечной дисперсии инноваций и, более того, при условиях, влекущих локальную асимптотическую нормальность (ЛАН) наблюдений. Знаково-ранговые аналоги этих оптимальных процедур были получены в [12] при дополнительном предположении о симметрии $G$. Знаковые автокорреляции - частный случай знаково-ранговых автокорреляций из [12].

В разделе 3 мы возвращаемся к стационарной модели (1.1) и рассматриваем для этой модели знаковые статистики из раздела 2. Наша цель - найти асимптотическую мощность знаковых тестов при близких альтернативах. Мы рассматриваем альтернативы, при которых параметры отличаются от гипотезы на $O\left(n^{-1 / 2}\right)$. Такие альтернативы не всегда являются локальными. Обычно термин «локальный» используют в асимптотической постановке в контексте локальных экспериментов, определенных при условии ЛАН, см., например, [20] и [23]. Наши альтернативы локальны в этом смысле, если модель (1.1) удовлетворяет условию ЛАН, т.е., в частности, при $\mathbf{E} \varepsilon_{1}^{2}<\infty$. (Напомним, информация Фишера о с, содержащаяся в $u_{1}, \ldots, u_{n}$, конечна при $\mathbf{E} \varepsilon_{1}^{2}<\infty$, см., например, [18] для ЛАН в ARMA модели.) Для ранговых, знаковоранговых и классических статистик в ARMA модели локальная мощность и свойства асимптотической локальной оптимальности были исследованы сравнительно недавно при условии ЛАН с помощью третьей леммы Ле Кама. Наш подход иной. Мы устанавливаем асимптотическую равномерную линейность (АРЛ) знаковых тестовых статистик и, как следствие, находим мощность тестов при близких альтернативах. Асимптотическая равномерная линейность статистик, основанных на остатках, уже давно изучается в связи с задачами оценивания и про- 
верки гипотез, см. [17]. Наш АРЛ-результат использует подход, развитый в [3]. Мы находим также асимптотическую относительную эффективность знаковых тестов относительно ранговых и классических тестов. Для функции распределения $G$ с тяжелыми хвостами она может быть сколь угодно больше единицы.

В разделе 4 мы строим знаковые тесты для линейных гипотез относительно с и находим их асимптотическую мощность. Для квадратических ранговых, знаково-ранговых и классических тестов соответствующие результаты (т.е. возможность замены мешающих параметров на $n^{1 / 2}$-состоятельные оценки, вычисление локальных мощностей и свойства асимптотической локальной оптимальности) получены опять не так давно при условии ЛАН, см. [13], [14], [19]. Наш результат для линейных гипотез - следствие АРЛ знаковых статистик.

Наконец отметим, что знаковые тесты в $\mathrm{AR}(p)$ модели изучались в [3]. В настоящей работе мы рассматриваем общую ARMA модель и при более слабых (в частности, моментных) ограничениях на $G$.

Доказательства теорем вынесены в раздел 5 .

2. Локально наиболее мошные знаковые тесты для выборок конечного объема. В этом разделе мы рассматриваем уравнение (1.1) при $t=1, \ldots, n$ и нулевых начальных условиях $u_{1-p}=\cdots=u_{0}=0=$ $\varepsilon_{1-q}=\cdots=\varepsilon_{0}$. Случайные величины $\left\{\varepsilon_{t}\right\}, t \geqslant 1$, независимы и одинаково распределены с функцией распределения $G$. Пусть $u_{1}, \ldots, u_{n}-$ соответствующие наблюдения. Мы построим знаковые тесты для проверки гипотез относительно вектора с при конечных $n$. Эти результаты необходимы в разделах 3 и 4 при рассмотрении основной стационарной модели (1.1).

Наши тесты будут основываться на знаках остатков, получаемых следующим образом. Для каждого $\boldsymbol{\theta}^{T}=\left(\theta_{1}, \ldots, \theta_{p+q}\right) \in \mathbf{R}^{p+q}$, определим рекуррентно

$$
\varepsilon_{t}(\boldsymbol{\theta})=u_{t}-\theta_{1} u_{t-1}-\cdots-\theta_{p} u_{t-p}-\theta_{p+1} \varepsilon_{t-1}(\boldsymbol{\theta})-\cdots-\theta_{p+q} \varepsilon_{t-q}(\boldsymbol{\theta}),
$$

с $\varepsilon_{1-q}(\boldsymbol{\theta})=\varepsilon_{2-q}(\boldsymbol{\theta})=\cdots=\varepsilon_{0}(\boldsymbol{\theta})=0, t=1, \ldots, n$. Положим

$$
S_{t}(\boldsymbol{\theta})=\operatorname{sign} \varepsilon_{t}(\boldsymbol{\theta}), \quad \mathbf{S}^{n}(\boldsymbol{\theta})=\left(S_{1}(\boldsymbol{\theta}), \ldots, S_{n}(\boldsymbol{\theta})\right) .
$$

Наша цель - проверить гипотезу

$$
H_{0}: \mathbf{c}=\mathbf{c}_{0},
$$

где $\mathbf{c}_{0}^{T}=\left(c_{10}, \ldots, c_{p+q, 0}\right)$ - полностью известный вектор, с помощью статистик, основанных на $\mathbf{S}^{n}\left(\mathbf{c}_{0}\right)$. Прежде всего мы хотим построить локально наиболее мощные (ЛНМ) тесты для $H_{0}$ при одномерных альтернативах

$$
H_{r}^{+}: \mathbf{c}=\mathbf{c}_{0}^{r}, \quad c_{r}>c_{r 0}, \quad r=1, \ldots, p+q,
$$


где $\mathbf{c}_{0}^{r}=\left(c_{10}, \ldots, c_{r-1,0}, c_{r}, c_{r+1,0}, \ldots, c_{p+q, 0}\right)^{T}$ - вектор с фиксированными для $i \neq r$ компонентами.

Предположим, что выполнены следующие условия.

У сло в и е (i): $\mathbf{P}\left\{\varepsilon_{1}<0\right\}=\mathbf{P}\left\{\varepsilon_{1}>0\right\}=\frac{1}{2}$.

У сл о в и е (ii): $\mathbf{E}\left|\varepsilon_{1}\right|<\infty, \mathbf{E} \varepsilon_{1}=0$.

У с л о в и е (iii): Существует $g=G^{\prime}, g(0)>0, \sup _{x} g(x)<\infty$, $g$ непрерывна в нуле.

Легко проверить, что при гипотезе $H_{0}$ имеем $\varepsilon_{t}\left(\mathbf{c}_{0}\right)=\varepsilon_{t}, t=$ $1, \ldots, n$. Поэтому если выполняются гипотеза $H_{0}$ и условие (i), то $S_{1}\left(\mathbf{c}_{0}\right), \ldots, S_{n}\left(\mathbf{c}_{0}\right)$ независимы, $\mathbf{P}_{c_{0}}\left\{S_{t}\left(\mathbf{c}_{0}\right)=1\right\}=\frac{1}{2}=\mathbf{P}_{c_{0}}\left\{S_{t}\left(\mathbf{c}_{0}\right)=-1\right\}$, $t=1, \ldots, n$, и, следовательно, наши тесты будут распределены свободно для конечных $n$.

Для $\mathbf{a}^{T}=\left(a_{1}, \ldots, a_{p}\right)$ определим последовательность $\left\{\alpha_{t}=\alpha_{t}(\mathbf{a})\right\}$ рекуррентным соотношением

$$
\alpha_{t}=a_{1} \alpha_{t-1}+\cdots+a_{p} \alpha_{t-p}, \quad t=1,2, \ldots,
$$

где $\alpha_{t}=0$ для $t<0$ и $\alpha_{0}=1$. Для $\mathbf{b}^{T}=\left(b_{1}, \ldots, b_{q}\right)$ определим также последовательность $\left\{\boldsymbol{\beta}_{t}=\beta_{t}(\mathbf{b})\right\}$ соотношением

$$
\beta_{t}=-b_{1} \beta_{t-1}-\cdots-b_{q} \beta_{t-q}, \quad t=1,2, \ldots,
$$

где $\beta_{t}=0$ для $t<0$ и $\beta_{0}=1$.

$\mathrm{C}$ вероятностью единица реализациями вектора $\mathbf{S}^{n}\left(\mathbf{c}_{0}\right)$ являются векторы $\mathrm{s}^{n}=\left(s_{1}, \ldots, s_{n}\right)$ с $s_{t}= \pm 1, t=1, \ldots, n$. Для любого такого $\mathbf{s}^{n}$ положим

$$
\gamma_{t n}\left(\mathbf{s}^{n}\right)=\frac{1}{n-t} \sum_{k=t+1}^{n} s_{k-t} s_{k}, \quad t=1, \ldots, n-1 .
$$

Тогда $\gamma_{t n}\left(\mathbf{s}^{n}\right)$ может интерпретироваться как эмпирическая автокорреляция порядка $t$ последовательности знаков $s_{1}, \ldots, s_{n}$, а величина $\frac{1}{2}(n-$ $t)\left[1-\gamma_{t n}\left(\mathbf{s}^{n}\right)\right]$ - как число обобщенных серий длины $t$ (см. [7]).

Теорема 2.1 дает разложение вероятности $\mathbf{P}_{\mathbf{c}_{0}^{r}}\left\{\mathbf{S}^{n}\left(\mathbf{c}_{0}\right)=\mathbf{s}^{n}\right\}$ при альтернативе $H_{r}^{+}$при $c_{r} \rightarrow c_{r 0}$.

Теорема 2.1. Предположим, что $A R M A(p, q)$ модель с нулевьми начальными условиями справедлива. Тогда если выполнены условия (i)-(iii), mo

$$
\begin{aligned}
\mathbf{P}_{\mathbf{c}_{0}^{i}}\left\{\mathbf{S}^{n}\left(\mathbf{c}_{0}\right)=\mathbf{s}^{n}\right\}= & \left(\frac{1}{2}\right)^{n}\left[1+2 g(0) \mathbf{E}\left|\varepsilon_{1}\right|\right. \\
& \left.\times \sum_{t=0}^{n-i-1} \alpha_{t}\left(\mathbf{a}_{0}\right)(n-t-i) \gamma_{t+i, n}\left(\mathbf{s}^{n}\right)\left(a_{i}-a_{i 0}\right)\right] \\
& +o\left(a_{i}-a_{i 0}\right), \quad a_{i} \rightarrow a_{i 0}, \quad i=1, \ldots, p
\end{aligned}
$$




$$
\begin{aligned}
\mathbf{P}_{\mathbf{c}_{0}^{p+j}}\left\{\mathbf{S}^{n}\left(\mathbf{c}_{0}\right)=\mathbf{s}^{n}\right\}= & \left(\frac{1}{2}\right)^{n}\left[1+2 g(0) \mathbf{E}\left|\varepsilon_{1}\right|\right. \\
& \left.\times \sum_{t=0}^{n-j-1} \beta_{t}\left(\mathbf{b}_{0}\right)(n-t-j) \gamma_{t+j, n}\left(\mathbf{s}^{n}\right)\left(b_{j}-b_{j 0}\right)\right] \\
& +o\left(b_{j}-b_{j 0}\right), \quad b_{j} \rightarrow b_{j_{0}}, \quad j=1, \ldots, q .
\end{aligned}
$$

Доказательство теоремы 2.1 аналогично доказательству теоремы 7.2.1 в [3]. Как непосредственное следствие теоремы 2.1 мы получаем ЛНМ тесты для гипотезы $H_{0}$ против гипотезы $H_{r}^{+}$при конечных $n$.

Теорема 2.2. Если выполнень условия теоремы 2.1, то

(а) для $i=1, \ldots, p$ u $n \geqslant i+1$ mест для $H_{0}$ против $H_{i}^{+}$с критической областью

$$
\left\{\mathrm{s}^{n}: \sum_{t=0}^{n-i-1} \alpha_{t}\left(\mathbf{a}_{0}\right)(n-t-i) \gamma_{t+i, n}\left(\mathbf{s}^{n}\right)>\text { const }\right\}
$$

является локально наиболее мощным среди тестов, основанных на $\mathbf{S}^{n}\left(\mathbf{c}_{0}\right)$

(б) $\partial л я j=1, \ldots, q u n \geqslant j+1$ meст для $H_{0}$ против $H_{p+j}^{+}$с критической областью

$$
\left\{\mathbf{s}^{n}: \sum_{t=0}^{n-j-1} \beta_{t}\left(\mathbf{b}_{0}\right)(n-t-j) \gamma_{t+j, n}\left(\mathbf{s}^{n}\right)>\text { const }\right\}
$$

является локально наиболее мошньм среди тестов, основанньх Ha $\mathbf{S}^{n}\left(\mathbf{c}_{0}\right)$.

Похожие результаты справедливы для тестов, предназначенных для проверки гипотезы $H_{0}$ против $H_{r}^{-}$. Достаточно изменить в (2.6) и $(2.7)$ знаки неравенств на обратные.

Для $\boldsymbol{\theta}^{T}=\left(\boldsymbol{\theta}_{1}^{T}, \boldsymbol{\theta}_{2}^{T}\right), \boldsymbol{\theta}_{1}^{T}=\left(\theta_{1}, \ldots, \theta_{p}\right), \boldsymbol{\theta}_{2}^{T}=\left(\theta_{p+1}, \ldots, \theta_{p+q}\right)$ положим

$$
\Gamma_{t n}(\boldsymbol{\theta})=\frac{1}{n-t} \sum_{k=t+1}^{n} S_{k-t}(\boldsymbol{\theta}) S_{k}(\boldsymbol{\theta}), \quad t=1, \ldots, n-1 .
$$

Это знаковая автокорреляция порядка $t$. Положим также

$$
\begin{aligned}
& l_{i n}^{\mathbf{a}}(\boldsymbol{\theta})=\sum_{t=0}^{n-i-1} \alpha_{t}\left(\boldsymbol{\theta}_{1}\right)(n-t-i) \Gamma_{t+i, n}(\boldsymbol{\theta}), \quad i=1, \ldots, p, \\
& l_{j n}^{\mathbf{b}}(\boldsymbol{\theta})=\sum_{t=0}^{n-j-1} \beta_{t}\left(\boldsymbol{\theta}_{2}\right)(n-t-j) \Gamma_{t+j, n}(\boldsymbol{\theta}), \quad j=1, \ldots, q, \\
& \mathbf{l}_{n}^{S}(\boldsymbol{\theta})=\left(l_{1 n}^{\mathbf{a}}(\boldsymbol{\theta}), \ldots, l_{p n}^{\mathbf{a}}(\boldsymbol{\theta}), l_{1 n}^{\mathbf{b}}(\boldsymbol{\theta}), \ldots, l_{q n}^{\mathbf{b}}(\boldsymbol{\theta})\right)^{T} .
\end{aligned}
$$

Статистики $l_{i n}^{\mathbf{a}}\left(\mathbf{c}_{0}\right)$ и $l_{j n}^{\mathbf{b}}\left(\mathbf{c}_{0}\right)$ являются тестовыми для $(2.6)$ и $(2.7)$. 
Очевидно,

$$
\Gamma_{t n}(\mathbf{c})=\frac{1}{n-t} \sum_{k=t+1}^{n} S_{k-t}(\mathbf{c}) S_{k}(\mathbf{c})=\frac{1}{n-t} \sum_{k=t+1}^{n} \operatorname{sign}\left(\varepsilon_{k-t} \varepsilon_{k}\right) .
$$

При условии (i) распределение последней величины свободно от $G$. Следовательно, распределения $l_{i n}^{\mathbf{a}}\left(\mathbf{c}_{0}\right)$ и $l_{j n}^{\mathbf{b}}\left(\mathbf{c}_{0}\right)$ также свободны от $G$ для каждого $n$ при гипотезе $H_{0}$ и условии $(\mathrm{i})$. Для данных $\mathbf{a}_{0}$ и $\mathbf{b}_{0}$ эти распределения могут быть вычислены методом Монте-Карло.

При условии (i) случайные переменные $\operatorname{sign}\left(\varepsilon_{1} \varepsilon_{t+1}\right), \ldots, \operatorname{sign}\left(\varepsilon_{n-t} \varepsilon_{n}\right)$ независимы,

$$
\mathbf{P}\left\{\operatorname{sign}\left(\varepsilon_{k-t} \varepsilon_{k}\right)=-1\right\}=\frac{1}{2}=\mathbf{P}\left\{\operatorname{sign}\left(\varepsilon_{k-t} \varepsilon_{k}\right)=1\right\}, \quad k=t+1, \ldots, n,
$$

см. [6]. Следовательно, при гипотезе $H_{0}$ и условии (i) величина

$$
\frac{1}{2} \sum_{k=t+1}^{n}\left[1+\operatorname{sign}\left(\varepsilon_{k-t} \varepsilon_{k}\right)\right]=\frac{1}{2}\left[n-t+(n-t) \Gamma_{t n}\left(\mathbf{c}_{0}\right)\right]
$$

имеет биномиальное распределение $\operatorname{Bin}\left(n-t, \frac{1}{2}\right)$. Кроме того, очевидно, что при условии (i)

$$
\mathbf{E}_{\mathbf{c}} \Gamma_{t n}(\mathbf{c})=0 \quad \text { и } \quad \mathbf{E}_{\mathbf{c}} \Gamma_{t n}(\mathbf{c}) \Gamma_{r n}(\mathbf{c})=(n-t)^{-1} \delta_{t r}
$$

где $\delta_{t r}$ - символ Кронекера. С помощью (2.12) легко получаем, что ковариационная матрица вектора $\mathbf{l}_{n}^{S}(\mathbf{c})$ при условии (i) равна

$$
\mathbf{K}_{n}(\mathbf{c})=\left(\begin{array}{ll}
\mathbf{A}_{n}(\mathbf{a}) & \boldsymbol{\Omega}_{n}(\mathbf{c}) \\
\boldsymbol{\Omega}_{n}^{T}(\mathbf{c}) & \mathbf{B}_{n}(\mathbf{b})
\end{array}\right)
$$

где $\mathbf{A}_{n}(\mathbf{a})=\left(a_{i j}^{n}(\mathbf{a})\right)_{1 \leqslant i, j \leqslant p}, \quad \mathbf{B}_{n}(\mathbf{b})=\left(b_{i j}^{n}(\mathbf{b})\right)_{1 \leqslant i, j \leqslant q}, \quad \boldsymbol{\Omega}_{n}(\mathbf{c})=$ $\left(\omega_{i j}^{n}(\mathbf{c})\right)_{\substack{i=1, \ldots, p \\ j=1, \ldots, q}} ;$ и

$$
\begin{aligned}
& a_{i j}^{n}(\mathbf{a})=\sum_{t=0}^{n-\max (i, j)-1} \alpha_{t} \alpha_{t+|i-j|}(n-t-\max (i, j)), \\
& b_{i j}^{n}(\mathbf{b})=\sum_{t=0}^{n-\max (i, j)-1} \beta_{t} \beta_{t+|i-j|}(n-t-\max (i, j)), \\
& \omega_{i j}^{n}(\mathbf{c})= \begin{cases}\sum_{t=0}^{n-j-1} \beta_{t} \alpha_{t+j-i}(n-t-j), & j \geqslant i, \\
\sum_{t=0}^{n-i-1} \alpha_{t} \beta_{t+i-j}(n-t-i), & j<i .\end{cases}
\end{aligned}
$$

Введем следующие стандартные усповия (см. [4, § 3.1]).

У с л о в и е (iv): Характеристические полиномы

$$
A(z)=z^{p}-a_{1} z^{p-1}-\cdots-a_{p} \text { и } B(z)=z^{q}+b_{1} z^{q-1}+\cdots+b_{q}
$$

не имеют корней при $|z| \geqslant 1$. 
У с л о в и е $(\mathrm{v}): A(z), B(z)$ не имеют общих корней.

При условии (iv) $\alpha_{t}$ и $\beta_{t}$ стремятся к нулю с экспоненциальной скоростью, так что существует предел

$$
\mathbf{K}(\mathbf{c}):=\lim n^{-1} \mathbf{K}_{n}(\mathbf{c}) .
$$

Если кроме условия (iv) выполнено условие (v), то $K(\mathbf{c})>0$.

В силу теоремы 2.1

$$
\begin{aligned}
& \left.\frac{\partial \log \mathbf{P}_{\mathbf{c}_{0}^{i}}\left\{\mathbf{S}^{n}\left(\mathbf{c}_{0}\right)=\mathbf{s}^{n}\right\}}{\partial c_{i}}\right|_{c_{i}=c_{i 0}} \\
& =2 g(0) \mathbf{E}\left|\varepsilon_{1}\right| \sum_{t=0}^{n-i-1} \alpha_{t}\left(\mathbf{a}_{0}\right)(n-t-i) \gamma_{t+i, n}\left(\mathbf{s}^{n}\right), \\
& \left.\frac{\partial \log \mathbf{P}_{\mathbf{c}_{0}^{p+j}}\left\{\mathbf{S}^{n}\left(\mathbf{c}_{0}\right)=\mathbf{s}^{n}\right\}}{\partial c_{p+j}}\right|_{c_{p+j}=c_{p+j, 0}} \\
& =2 g(0) \mathbf{E}\left|\varepsilon_{1}\right| \sum_{t=0}^{n-j-1} \beta_{t}\left(\mathbf{b}_{0}\right)(n-t-j) \gamma_{t+j, n}\left(\mathbf{s}^{n}\right) .
\end{aligned}
$$

Пусть $\mathbf{I}_{n}(\mathbf{c}, \boldsymbol{\theta})$ - информация Фишера о векторе с, основанная на $\mathbf{S}^{n}(\boldsymbol{\theta})$. В силу $(2.14)$ и $(2.15)$

$$
\mathbf{I}_{n}(\mathbf{c}, \mathbf{c})=\left(2 g(0) \mathbf{E}\left|\varepsilon_{1}\right|\right)^{2} \mathbf{E}_{c} \mathbf{l}_{n}^{S}(\mathbf{c})\left(\mathbf{l}_{n}^{S}(\mathbf{c})\right)^{T}=\left(2 g(0) \mathbf{E}\left|\varepsilon_{1}\right|\right)^{2} \mathbf{K}_{n}(\mathbf{c}) .
$$

При условии $(\mathrm{v}) \mathbf{K}_{n}(\mathbf{c})>0$, для любого $n \geqslant p+q+1$, следовательно, $\mathbf{I}_{n}(\mathbf{c}, \mathbf{c})>0$. При условиях (i) и (iv) в силу (2.13)

$$
\mathbf{I}_{n}(\mathbf{c}, \mathbf{c}) \sim\left(2 g(0) \mathbf{E}\left|\varepsilon_{1}\right|\right)^{2} n \mathbf{K}(\mathbf{c})
$$

Соотношения $(2.14),(2.15)$ и определения (2.8)-(2.11) позволяют предложить естественную квадратическую форму в качестве тестовой статистики для проверки гипотезы $H_{0}$ против $H_{1}: \mathbf{c} \neq \mathbf{c}_{0}$ :

$$
L_{n}^{S}\left(\mathbf{c}_{0}\right)=\left(2 g(0) \mathbf{E}\left|\varepsilon_{1}\right|\right)^{2}\left(\mathbf{l}_{n}^{S}\left(\mathbf{c}_{0}\right)\right)^{T} \mathbf{I}_{n}^{-1}\left(\mathbf{c}_{0}, \mathbf{c}_{0}\right) \mathbf{l}_{n}^{S}\left(\mathbf{c}_{0}\right)
$$

В силу (2.16)

$$
L_{n}^{S}\left(\mathbf{c}_{0}\right)=\left(\mathbf{l}_{n}^{S}\left(\mathbf{c}_{0}\right)\right)^{T} \mathbf{K}_{n}^{-1}\left(\mathbf{c}_{0}\right) \mathbf{l}_{n}^{S}\left(\mathbf{c}_{0}\right)
$$

При условии (i) нулевое распределение $L_{n}^{S}\left(\mathbf{c}_{0}\right)$ свободно для фиксированных $n$. Асимптотическое нулевое распределение $\mathbf{l}_{n}^{S}\left(\mathbf{c}_{0}\right)$ и $L_{n}^{S}\left(\mathbf{c}_{0}\right)$ найти легко. При условиях (i), (iv) и $H_{0}$ имеет место сходимость по распределению

$$
n^{-1 / 2} \mathbf{l}_{n}^{S}\left(\mathbf{c}_{0}\right) \stackrel{d_{\mathbf{c}_{0}}}{\longrightarrow} \mathscr{N}\left(0, K\left(\mathbf{c}_{0}\right)\right) .
$$

Соотношение (2.19) доказывается аналогично теореме 7.2 .4 в [3]. Если кроме условий (i) и (iv) выполнено условие (v), то $K\left(\mathbf{c}_{0}\right)>0$, и в силу (2.13), (2.19)

$$
L_{n}^{S}\left(\mathbf{c}_{0}\right) \stackrel{d_{\mathbf{c}_{0}}}{\longrightarrow} \chi^{2}(p+q)
$$

$\chi^{2}(p+q)$ - распределение хи-квадрат с $p+q$ степенями свободы. 
3. Мощность знаковых тестов при близких альтернативах. В этом разделе мы рассмотрим знаковые статистики из раздела 2 в стационарной модели (1.1) при условиях (i)-(v). При условиях (ii), (iv) уравнение (1.1) имеет единственное строго стационарное решение. Пусть $u_{1-p}, \ldots, u_{n}$ есть наблюдения из этого решения. Пусть вектор $\mathbf{l}_{n}^{S}(\boldsymbol{\theta})$ определен соотношениями $(2.8)-(2.11)$ с остатками $\varepsilon_{t}(\boldsymbol{\theta})$ и знаками $S_{t}(\boldsymbol{\theta})$ из (2.1) и (2.2). Наша цель - найти асимптотическое распределение знаковой статистики $L_{n}^{S}\left(\mathbf{c}_{0}\right)$ из (2.18) при гипотезе $H_{0}: \mathbf{c}=\mathbf{c}_{0}$ из $(2.3)$ и близких альтернативах

$$
H_{1 n}(\mathbf{d}): \mathbf{c}=\mathbf{c}_{n}:=\mathbf{c}_{0}+\mathbf{d} n^{-1 / 2}+o\left(n^{-1 / 2}\right), \quad \mathbf{d} \in \mathbf{R}^{p+q},
$$

где $\mathbf{d}$ - постоянный вектор. Поскольку при условии (ii) дисперсия $\mathbf{E} \varepsilon_{1}^{2}$, вообще говоря, бесконечна, то и информация Фишера о векторе с, возможно, бесконечна. В такой ситуации условие ЛАН не выполнено и использовать обычный способ - третью лемму Ле Кама - для отыскания предельного распределения $L_{n}^{S}\left(\mathbf{c}_{0}\right)$ при $H_{1 n}(\mathbf{d})$ невозможно. Мы воспользуемся другим подходом: установим асимптотическую равномерную линейность процесса $n^{-1 / 2} \mathbf{l}_{n}^{S}\left(\mathbf{c}_{n}+n^{-1 / 2} \boldsymbol{\tau}\right), \boldsymbol{\tau} \in \mathbf{R}^{p+q}$. В следующем разделе этот результат позволит построить знаковые тесты для линейных гипотез.

$$
\begin{gathered}
\text { Положим } \widetilde{\Gamma}_{t n}:=(n-t)^{-1} \sum_{k=t+1}^{n} \operatorname{sign}\left(\varepsilon_{k-t} \varepsilon_{k}\right), t=1, \ldots, n-1, \\
\begin{cases}\tilde{l}_{i n}^{\mathbf{a}}\left(\mathbf{a}_{0}\right):=\sum_{t=0}^{n-i-1} \alpha_{t}\left(\mathbf{a}_{0}\right)(n-t-i) \widetilde{\Gamma}_{t+i, n}, & i=1, \ldots, p, \\
\tilde{l}_{j n}^{\mathbf{b}}\left(\mathbf{b}_{0}\right):=\sum_{t=0}^{n-j-1} \beta_{t}\left(\mathbf{b}_{0}\right)(n-t-j) \widetilde{\Gamma}_{t+j, n}, & j=1, \ldots, q,\end{cases}
\end{gathered}
$$

последовательности $\left\{\alpha_{t}\right\}$ и $\left\{\beta_{t}\right\}$ определены в (2.4) и (2.5) соответственно. Пусть $\tilde{\mathbf{l}}_{n}^{S}\left(\mathbf{c}_{0}\right):=\left(\tilde{l}_{1 n}^{\mathbf{a}}\left(\mathbf{a}_{0}\right), \ldots, \tilde{l}_{p n}^{\mathbf{a}}\left(\mathbf{a}_{0}\right), \tilde{l}_{1 n}^{\mathbf{b}}\left(\mathbf{b}_{0}\right), \ldots, \tilde{l}_{q n}^{\mathbf{b}}\left(\mathbf{b}_{0}\right)\right)^{T}$, матрица $K(\mathbf{c})$ определена в $(2.13)$ и $\|\cdot\|$ обозначает евклидову норму вектора.

Теорема 3.1. Предположим, что верна ARMA модель (1.1) при условиях (i)-(iv). Тогда при альтернативе $H_{1 n}(\mathbf{d})$ для любого конечного $\Theta \geqslant 0$

$$
\sup _{\|\boldsymbol{\tau}\| \leqslant \Theta}\left\|n^{-1 / 2} \mathbf{l}_{n}^{S}\left(\mathbf{c}_{n}+n^{-1 / 2} \boldsymbol{\tau}\right)-n^{-1 / 2} \widetilde{\mathbf{l}}_{n}^{S}\left(\mathbf{c}_{0}\right)+2 g(0) \mathbf{E}\left|\varepsilon_{1}\right| \mathbf{K}\left(\mathbf{c}_{0}\right) \boldsymbol{\tau}\right\|=o_{\mathbf{P}}(1) .
$$

Доказательство этого результата вынесено в раздел 5. Из теоремы 3.1 стандартным образом вытекает следствие 3.1.

Следствие 3.1. Если выполняются условия теоремь 3.1, для любой последовательности $\left\{\widehat{\mathbf{c}}_{n}\right\}$ случайных векторов, удовлетворяюших условию $n^{1 / 2}\left(\widehat{\mathbf{c}}_{n}-\mathbf{c}_{n}\right)=O_{\mathbf{P}}(1)$,

$$
n^{-1 / 2} \mathbf{l}_{n}^{S}\left(\widehat{\mathbf{c}}_{n}\right)=n^{-1 / 2} \tilde{\mathbf{l}}_{n}^{S}\left(\mathbf{c}_{0}\right)-2 g(0) \mathbf{E}\left|\varepsilon_{1}\right| \mathbf{K}\left(\mathbf{c}_{0}\right) n^{1 / 2}\left(\widehat{\mathbf{c}}_{n}-\mathbf{c}_{n}\right)+o \mathbf{P}(1) .
$$


В силу (2.19) при условиях (i) и (iv)

$$
n^{-1 / 2} \widetilde{\mathbf{l}}_{n}^{S}\left(\mathbf{c}_{0}\right) \stackrel{d_{\mathbf{c}_{\mathfrak{n}}}}{\rightarrow} \mathscr{N}\left(0, \mathbf{K}\left(\mathbf{c}_{0}\right)\right)
$$

Этот факт, следствие 3.1 и соотношение $n^{1 / 2}\left(\mathbf{c}_{0}-\mathbf{c}_{n}\right)=-\mathbf{d}+o(1)$ влекут следующий результат, в котором $\chi^{2}\left(r, \lambda^{2}\right)$ обозначает нецентральное хи-квадрат распределение с $r$ степенями свободы и параметром нецентральности $\lambda^{2}$.

Следствие 3.2. Предположим, что справедлива модель (1.1) при условиях (i)-(iv). Тогда при $H_{1 n}(\mathbf{d})$

$$
n^{-1 / 2} \mathbf{l}_{n}^{S}\left(\mathbf{c}_{0}\right)=n^{-1 / 2} \tilde{\mathbf{l}}_{n}^{S}\left(\mathbf{c}_{0}\right)+2 g(0) \mathbf{E}\left|\varepsilon_{1}\right| \mathbf{K}\left(\mathbf{c}_{0}\right) \mathbf{d}+o \mathbf{p}(1)
$$

u, следовательно,

$$
n^{-1 / 2} \mathbf{l}_{n}^{S}\left(\mathbf{c}_{0}\right) \stackrel{d_{\mathbf{c}_{\mathbf{n}}}}{\longrightarrow} \mathscr{N}\left(2 g(0) \mathbf{E}\left|\varepsilon_{1}\right| \mathbf{K}\left(\mathbf{c}_{0}\right) \mathbf{d}, \mathbf{K}\left(\mathbf{c}_{0}\right)\right) .
$$

Если, кроме того выполнено условие (v), то

$$
L_{n}^{S}\left(\mathbf{c}_{0}\right) \stackrel{d_{\mathbf{c}_{n}}}{\longrightarrow} \chi^{2}\left(p+q, \lambda_{S}^{2}\right), \quad \lambda_{S}^{2}=\left(2 g(0) \mathbf{E}\left|\varepsilon_{1}\right|\right)^{2} \mathbf{d}^{T} \mathbf{K}\left(\mathbf{c}_{0}\right) \mathbf{d} .
$$

При аипотезе $H_{0}$ имеем $L_{n}^{S}\left(\mathbf{c}_{0}\right) \stackrel{d_{\mathbf{c}_{0}}}{\longrightarrow} \chi^{2}(p+q)$.

Следствие 3.2 позволяет находить асимптотическую относительную эффективность (АОЭ) знаковых тестов относительно известных тестов. Сейчас мы сравним знаковые тесты с квадратическими ранговыми тестами. Они были предложены и детально изучены в [10] и [11] при условиях, влекущих ЛАН. Знаково-ранговые аналоги этих тестов были изучены в [12]. Результаты суммированы в обзоре [13].

Пусть $f$ обозначает плотность вероятности, соответствующую функции распределения $F$. Положим $\phi_{f}:=-f^{\prime} / f$. Пусть

$$
r_{t n, f}(\boldsymbol{\theta}):=\left[\frac{1}{n-t} \sum_{k=t+1}^{n} F^{-1}\left(\frac{R_{k-t}(\boldsymbol{\theta})}{n+1}\right) \phi_{f}\left(F^{-1}\left(\frac{R_{k}(\boldsymbol{\theta})}{n+1}\right)\right)-m_{f}^{(n)}\right] / \sigma_{t, f}^{(n)} .
$$

Статистика $r_{t n, f}(\boldsymbol{\theta})$ называется ранговой $f$-автокорреляцией порядка $t$. В (3.2) через $R_{t}(\boldsymbol{\theta})$ обозначен ранг остатка $\varepsilon_{t}(\boldsymbol{\theta})$ в последовательности $\varepsilon_{1}(\boldsymbol{\theta}), \ldots, \varepsilon_{n}(\boldsymbol{\theta})$, а $m_{f}^{(n)}$ и $\sigma_{t, f}^{(n)}$ определены соотношениями (3.6) и (3.7) в [13], их явный вид здесь не важен.

Пусть $\psi:=\left\{\psi_{t}^{(1)}, \ldots, \psi_{t}^{(p+q)}\right\}$ будет любая фундаментальная система решений однородного уравнения $A(L) B(L) \psi_{t}=0, t \in \mathbf{Z}$, в котором $L-$ оператор запаздывания; $A(\cdot), B(\cdot)$ - характеристические полиномы из условия (iv). Рассмотрим вектор

$$
\begin{aligned}
\mathbf{l}_{n, f, \boldsymbol{\psi}}^{R}\left(\mathbf{c}_{0}\right):= & \left(\sum_{t=1}^{n-1}(n-t)^{1 / 2} \psi_{t}^{(1)}\left(\mathbf{c}_{0}\right) r_{t n, f}\left(\mathbf{c}_{0}\right), \ldots,\right. \\
& \left.\sum_{t=1}^{n-1}(n-t)^{1 / 2} \psi_{t}^{(p+q)}\left(\mathbf{c}_{0}\right) r_{t n, f}\left(\mathbf{c}_{0}\right)\right)^{T},
\end{aligned}
$$


и $(p+q) \times(p+q)$ матрицу

$$
\mathbf{K}_{\psi}\left(\mathbf{c}_{0}\right):=\left(k_{\psi, i j}\left(\mathbf{c}_{0}\right)\right), \quad k_{\psi, i j}\left(\mathbf{c}_{0}\right)=\sum_{t \geqslant 1} \psi_{t}^{(i)}\left(\mathbf{c}_{0}\right) \psi_{t}^{(j)}\left(\mathbf{c}_{0}\right) .
$$

Квадратическая ранговая тестовая статистика для гипотезы $H_{0}$ имеет вид

$$
L_{n, f, \psi}^{R}\left(\mathbf{c}_{0}\right):=\left(\mathbf{l}_{n, f, \psi}^{R}\left(\mathbf{c}_{0}\right)\right)^{T} \mathbf{K}_{\psi}^{-1}\left(\mathbf{c}_{0}\right) \mathbf{l}_{n, f, \psi}^{R}\left(\mathbf{c}_{0}\right) .
$$

Если $f$ - нормальная, логистическая или двойная экспоненциальная плотность соответственно, то получаются известные статистики Ван дер Вардена, Вилкоксона и Лапласа.

В силу предложения 4.4 из [13] при альтернативе $H_{1 n}(\mathbf{d})$ статистика $L_{n, f, \psi}^{R}\left(\mathbf{c}_{0}\right)$ асимптотически распределена как $\chi^{2}\left(p+q-m, \lambda_{R}^{2}(f)\right)$. Статистика $L_{n, f, \psi}^{R}\left(\mathbf{c}_{0}\right)$ от фундаментальной системы не зависит, т.е.

$$
L_{n, f, \boldsymbol{\psi}}^{R}\left(\mathbf{c}_{0}\right)=L_{n, f, \boldsymbol{\psi}_{0}}^{R}\left(\mathbf{c}_{0}\right) .
$$

Следовательно, и параметр нецентральности не зависит от $\boldsymbol{\psi}$, его можно представить в виде

$$
\begin{gathered}
\lambda_{R}^{2}(f)=I^{2}(f \mid g) \mathbf{d}^{T} \mathbf{K}\left(\mathbf{c}_{0}\right) \mathbf{d} \\
I(f \mid g):=\left\{\int_{0}^{1} \phi_{f}\left(F^{-1}(u)\right) \phi_{g}\left(G^{-1}(u)\right) d u \int_{0}^{1} F^{-1}(u) G^{-1}(u) d u\right\} \\
\times\left\{\mathbf{E} \varepsilon_{1}^{2} i(g)\right\}^{-1 / 2},
\end{gathered}
$$

где $i(g)$ - информация Фишера плотности $g$.

При $f=g$ тест со статистикой (3.4) является асимптотически локально максиминным (см. предложение 4.3 в [11] для уточнений альтернативы и условий на $g$ ).

В силу (3.5) и следствия 3.2 асимптотическая относительная эффективность знакового теста со статистикой $L_{n}^{S}\left(\mathbf{c}_{0}\right)$ относительно рангового теста со статистикой $L_{n, f, \psi}^{R}\left(\mathbf{c}_{0}\right)$ равна

$$
e_{S, R}(f)=\frac{\left(2 g(0) \mathbf{E}\left|\varepsilon_{1}\right|\right)^{2}}{I^{2}(f \mid g)} .
$$

Эта АОЭ не зависит от вектора с и $_{0}$ и параметров масштаба $G$ и $F$.

Поскольку $I^{2}(f \mid g) \leqslant i(f) \int_{-\infty}^{\infty} x^{2} d F(x)$, то

$$
e_{S, R}(f) \geqslant \frac{e_{S}}{i(f) \int_{-\infty}^{\infty} x^{2} d F(x)}, \quad \text { где } \quad e_{S}:=\left(2 g(0) \mathbf{E}\left|\varepsilon_{1}\right|\right)^{2} .
$$

Для $G$ с тяжелыми хвостами $e_{s}$ может быть сколь угодно большой; в такой ситуации знаковые тесты предпочтительнее квадратических ранговых.

В [12] была установлена асимптотическая эквивалентность знаковоранговых и ранговых тестов. Следовательно, АОЭ знаковых тестов относительно знаково-ранговых опять равна $e_{S, R}(f)$ из $(3.7)$. 
Теперь рассмотрим классические тесты, основанные на обычных автокорреляциях остатков

$$
r_{t n}(\boldsymbol{\theta})=\frac{(n-t)^{-1} \sum_{k=t+1}^{n} \varepsilon_{k-t}(\boldsymbol{\theta}) \varepsilon_{k}(\boldsymbol{\theta})}{n^{-1} \sum_{k=1}^{n} \varepsilon_{k}^{2}(\boldsymbol{\theta})} .
$$

Пусть

$$
\begin{aligned}
\mathbf{l}_{n, \boldsymbol{\psi}}\left(\mathbf{c}_{0}\right):= & \left(\sum_{t=1}^{n-1}(n-t)^{1 / 2} \psi_{t}^{(1)}\left(\mathbf{c}_{0}\right) r_{t n}\left(\mathbf{c}_{0}\right), \ldots,\right. \\
& \left.\sum_{t=1}^{n-1}(n-t)^{1 / 2} \psi_{t}^{(p+q)}\left(\mathbf{c}_{0}\right) r_{t n}\left(\mathbf{c}_{0}\right)\right)^{T}, \\
L_{n, \boldsymbol{\psi}}\left(\mathbf{c}_{0}\right):= & \left(\mathbf{l}_{n, \boldsymbol{\psi}}\left(\mathbf{c}_{0}\right)\right)^{T} \mathbf{K}_{\psi}^{-1}\left(\mathbf{c}_{0}\right) \mathbf{l}_{n, \psi}\left(\mathbf{c}_{0}\right)
\end{aligned}
$$

Если инновации $\left\{\varepsilon_{t}\right\}$ гауссовские, то тест со статистикой $L_{n, \psi}\left(\mathbf{c}_{0}\right)$ является локально асимптотически максиминным (см. [13, предложение 5.4]). В общей ситуации при $H_{1 n}(\mathbf{d})$ асимптотическое распределение $L_{n, \psi}\left(\mathbf{c}_{0}\right)$ есть $\chi^{2}\left(p-m, \lambda^{2}\right)$,

$$
\lambda^{2}=\mathbf{d}^{T} \mathbf{K}\left(\mathbf{c}_{0}\right) \mathbf{d}
$$

В силу (3.10) и следствия 3.2 АОЭ знакового теста со статистикой $L_{n}^{S}\left(\mathbf{c}_{0}\right)$ относительно классического теста со статистикой $L_{n, \boldsymbol{\psi}}\left(\mathbf{c}_{0}\right)$ равна

$$
\left(2 g(0) \mathbf{E}\left|\varepsilon_{1}\right|\right)^{2}=e_{S}
$$

Для функции распределения $G$ с тяжелыми хвостами, когда эффективность $e_{s}$ велика, знаковые тесты предпочтительнее классических.

3 а м е ч а н и е 3.1 . Статистика $L_{n}^{S}\left(\mathbf{c}_{0}\right)$ допускает асимптотическое представление в виде, аналогичном (3.4) и (3.9). А именно, пусть

$$
\begin{aligned}
\overline{\mathbf{l}}_{n, \boldsymbol{\psi}}^{S}\left(\mathbf{c}_{0}\right):= & \left(\sum_{t=1}^{n-1}(n-t)^{1 / 2} \psi_{t}^{(1)}\left(\mathbf{c}_{0}\right) \Gamma_{t n}\left(\mathbf{c}_{0}\right), \ldots,\right. \\
& \left.\sum_{t=1}^{n-1}(n-t)^{1 / 2} \psi_{t}^{(p+q)}\left(\mathbf{c}_{0}\right) \Gamma_{t n}\left(\mathbf{c}_{0}\right)\right)^{T}, \\
\bar{L}_{n, \psi}^{S}\left(\mathbf{c}_{0}\right):= & \left(\overline{\mathbf{l}}_{n, \psi}^{S}\left(\mathbf{c}_{0}\right)\right)^{T} \mathbf{K}_{\psi}^{-1}\left(\mathbf{c}_{0}\right) \overline{\mathbf{l}}_{n, \psi}^{S}\left(\mathbf{c}_{0}\right) .
\end{aligned}
$$

Легко проверить, что $\bar{L}_{n}^{S}\left(\mathbf{c}_{0}\right)$ не зависит от $\psi$, и $L_{n}^{S}\left(\mathbf{c}_{0}\right)-\bar{L}_{n, \boldsymbol{\psi}}^{S}\left(\mathbf{c}_{0}\right)=$ $O\left(n^{-1 / 2}\right)$. При этом $\bar{L}_{n, \psi}^{S}\left(\mathbf{c}_{0}\right)$ получается из $L_{n, f, \psi}^{R}\left(\mathbf{c}_{0}\right)$ или $L_{n, \psi}\left(\mathbf{c}_{0}\right)$ простой заменой ранговых или обычных автокорреляций на знаковые. Разумеется, для $\bar{L}_{n, \boldsymbol{\psi}}^{S}\left(\mathbf{c}_{0}\right)$ справедливы все утверждения об асимптотическом поведении, верные для $L_{n}^{S}\left(\mathbf{c}_{0}\right)$.

3 а м е ч а н и е 3.2. Иногда условие (v) может быть слишком ограничительным. Например, оно не выполнено и матрица $\mathbf{K}\left(\mathbf{c}_{0}\right)$ вырождена 
при $a_{p 0}=b_{q 0}=0$. Это не позволяет проверять гипотезы о фиксированной $\operatorname{ARMA}(p-1, q-1)$ модели против $\operatorname{ARMA}(p, q)$. Подобные задачи решаются с помощью квадратических ранговых, знаково-ранговых или классических статистик (см., например, [13]). Знаковые аналоги этих процедур можно строить, заменяя формально ранговые, знаковоранговые или классические автокорреляции на знаковые. Асимптотические свойства таких знаковых тестов при альтернативах типа (3.1) легко исследуются с помощью теоремы 3.1 и ее следствий.

4. Проверка линейных гипотез. В этом разделе мы построим знаковые тесты для проверки линейных гипотез относительно вектора $\mathbf{c}=\left(c_{1}, \ldots, c_{p+q}\right)^{T}$ в стационарной модели (1.1) при условиях (i) $-(\mathrm{v})$ и исследуем асимптотическую мощность этих тестов при близких альтернативах. Проверим гипотезу

$$
H_{0}^{L}: c_{i}=c_{i 0}, i=i_{1}, \ldots, i_{p+q-m} ; 1 \leqslant i_{1}<i_{2}<\cdots<i_{p+q-m} \leqslant p+q,
$$

где $\left\{c_{i 0}\right\}$ известны, а оставшиеся компоненты $\left\{c_{i}, i \neq i_{1}, i_{2}, \ldots, i_{p+q-m}\right\}$ являются мешающими параметрами; $1 \leqslant m<p+q$. Альтернатива имеет Вид:

$$
H_{1 n}^{L}(\mathbf{d}): \begin{cases}c_{i}=c_{i n}:=c_{i 0}+d_{i} n^{-1 / 2}+o\left(n^{-1 / 2}\right), & i=i_{1}, \ldots, i_{p+q-m}, \\ c_{i}=c_{i n}:=c_{i}+d_{i} n^{-1 / 2}+o\left(n^{-1 / 2}\right), & i \neq i_{1}, \ldots, i_{p+q-m}\end{cases}
$$

где $\mathbf{d}=\left(d_{1}, \ldots, d_{p+q}\right)^{T} \in \mathbf{R}^{p+q}, \mathbf{d}$ - постоянный вектор.

Пусть вектор $\mathbf{c}_{0}$ получается из вектора $\mathbf{c}$ заменой $c_{i}$ на $c_{i 0}$ для $i=i_{1}, \ldots, i_{p+q-m}$. Положим $\mathbf{c}_{n}:=\left(c_{1 n}, \ldots, c_{p+q, n}\right)^{T}$, пусть $\widehat{c}_{n}$ - оценка $c$, $\widehat{\mathbf{c}}_{n}:=\left(\widehat{c}_{1 n}, \ldots, \widehat{c}_{p+q, n}\right)^{T}$, и пусть $n^{1 / 2}\left(\widehat{\mathbf{c}}_{n}-\mathbf{c}_{n}\right)=O_{\mathbf{P}}(1)$ при $H_{1 n}^{L}(\mathbf{d})$. Положим $\widehat{\mathbf{c}}_{n 0}$ равным вектору $\widehat{\mathbf{c}}_{n}$, в котором $\widehat{c}_{i n}, i=i_{1}, \ldots, i_{p+q-m}$, заменены на $c_{i 0}$.

Пусть матрица $\mathrm{U}$ порядка $(p+q) \times(p+q)$ получается из единичной матрицы $\mathbf{I}_{p+q}$ какой-нибудь перестановкой строк с номерами $i_{1}, \ldots, i_{p+q-m}$ с последними $p+q-m$ строками. Тогда $\mathbf{U}=\mathbf{U}^{T}$, $\mathbf{U}=\mathbf{U}^{-1}$. Определим матрицы $\mathbf{J}_{\mathbf{U}}(\mathbf{c})$ порядка $m \times m, \mathbf{M}_{\mathbf{U}}(\mathbf{c})$ порядка $(p+q-m) \times(p+q-m)$ и $\mathbf{B}_{\mathbf{U}}^{T}(\mathbf{c})$ порядка $m \times(p+q-m)$ равенством

$$
\mathbf{U K}(\mathbf{c}) \mathbf{U}=\left(\begin{array}{ll}
\mathbf{J}_{\mathbf{U}}(\mathbf{c}) & \mathbf{B}_{\mathbf{U}}^{T}(\mathbf{c}) \\
\mathbf{B}_{\mathbf{U}}(\mathbf{c}) & \mathbf{M}_{\mathbf{U}}(\mathbf{c})
\end{array}\right)
$$

с матрицей $\mathbf{K}(\mathbf{c})$ из (2.13). Пусть

$$
\begin{aligned}
& \mathbf{W}_{\mathbf{U}}(\mathbf{c}):=\left(\begin{array}{cc}
\mathbf{J}_{\mathbf{U}}^{-1 / 2}(\mathbf{c}) & -\mathbf{J}_{\mathbf{U}}^{-1}(\mathbf{c}) \mathbf{B}_{\mathbf{U}}^{T}(\mathbf{c}) \mathbf{C}_{\mathbf{U}}^{-1 / 2}(\mathbf{c}) \\
\mathbf{0} & \mathbf{C}_{\mathbf{U}}^{-1 / 2}(\mathbf{c})
\end{array}\right) \\
& \mathbf{C}_{\mathbf{U}}(\mathbf{c}):=\mathbf{M}_{\mathbf{U}}(\mathbf{c})-\mathbf{B}_{\mathbf{U}}(\mathbf{c}) \mathbf{J}_{\mathbf{U}}^{-1}(\mathbf{c}) \mathbf{B}_{\mathbf{U}}^{T}(\mathbf{c})
\end{aligned}
$$

Все обратные матрицы определены, так как $\mathbf{K}(\mathbf{c})>0$. Обозначим $\widehat{\mathbf{W}}_{n, \mathbf{U}}$ состоятельную при $H_{1 n}^{L}(\mathbf{d})$ оценку матрицы $\mathbf{W}_{\mathbf{U}}\left(\mathbf{c}_{0}\right)$, например, можно положить $\widehat{\mathbf{W}}_{n, \mathbf{U}}=\mathbf{W}_{\mathbf{U}}\left(\widehat{\mathbf{c}}_{n}\right)$. 
В качестве тестовой статистики для $H_{0}^{L}$ возьмем

$$
\widehat{L}_{n}^{S}\left(\mathbf{U}, \widehat{\mathbf{c}}_{n 0}\right):=n^{-1}\left\|\pi \circ \widehat{\mathbf{W}}_{n, \mathbf{U}}^{T} \mathbf{U l}_{n}^{S}\left(\widehat{\mathbf{c}}_{n 0}\right)\right\|^{2},
$$

где $\pi$-ортогональная проекция на последние $p+q-m$ координат, вектор $\mathbf{l}_{n}^{S}(\cdot)$ из (2.11).

3 а м е ч а н и е 4.1. Объясним, почему тестовая статистика имеет такой вид. Предположим, что модель (1.1) удовлетворяет условиям ЛАН, и пусть $\boldsymbol{\Delta}_{n}(\mathbf{c})$ и $\mathscr{E}^{(n)}(\mathbf{c})=\left\{\mathbf{P}_{\mathbf{c}+n^{-1 / 2} \mathbf{d}}, \mathbf{d} \in \mathbf{R}^{p+q}\right\}$ будут асимптотически гауссовская центральная последовательность и последовательность локальных экспериментов (см. [18]). Если центр локализации $\mathbf{c}=\mathbf{c}_{0}$, то локальный вариант гипотезы $H_{0}^{L}$ из $(4.1)$ имеет вид

$$
H^{L}: \mathbf{d} \in \mathbf{L}, \quad \mathbf{L}:=\left\{\mathbf{x} \in \mathbf{R}^{p+q}: x_{i_{1}}=\cdots=x_{i_{p+q-m}}=0\right\} .
$$

Определим матрицу $\mathbf{W}_{\mathbf{U}}(\mathbf{c}, \Gamma)$ соотношениями (4.3)-(4.5), в которых матрица $\mathbf{K}(\mathbf{c})$ заменена на матрицу $\boldsymbol{\Gamma}(\mathbf{c}):=\mathbf{E} \varepsilon_{1}^{2} \mathbf{K}(\mathbf{c})$. Обозначим $\widehat{\mathbf{W}}_{n, \mathbf{U}}(\boldsymbol{\Gamma})$ состоятельную оценку $\mathbf{W}_{\mathbf{U}}\left(\mathbf{c}_{0}, \Gamma\right)$. Тогда тест для $H^{L}$ со статистикой

$$
\left\|\pi \circ \widehat{\mathbf{W}}_{n, \mathbf{U}}^{T}(\Gamma) \mathbf{U} \Delta_{n}\left(\widehat{\mathbf{c}}_{n 0}\right)\right\|^{2}
$$

будет асимптотически локально максиминным при альтернативе $K_{\delta}=$ $\left\{\mathbf{d} \in \mathbf{R}^{p+q}:\left\|\mathbf{d}-\pi_{L}(\mathbf{d})\right\|=\delta\right\}, \delta>0, \pi_{\mathbf{L}}-$ проекция на $\mathbf{L}$.

Доказательство этого факта аналогично доказательству теоремы 3.2 в [19], где были введены и изучены подобные асимптотически локально максиминные тесты для авторегрессионной модели.

Теперь видно, что знаковая статистика (4.6) есть аналог асимптотически локально оптимальной тестовой статистики (4.7).

Пусть вектор $\mathbf{d}_{\mathbf{U}}^{(2)}$ размера $p+q-m$ определяется соотношением $(\mathbf{U} \mathbf{d})^{T}=\left(\mathbf{d}_{\mathbf{U}}^{(1)^{T}}, \mathbf{d}_{\mathbf{U}}^{(2)^{T}}\right)$. Обозначим $\mathfrak{U}$ множество всех матриц $\mathbf{U}$.

Теорема 4.1. Пусть вьполнень условия (i)-(v). Тогда при гипотезе $H_{0}^{L}$

$$
\widehat{L}_{n}^{S}\left(\mathbf{U}, \widehat{\mathbf{c}}_{n 0}\right) \stackrel{d_{\mathbf{c}_{0}}}{\longrightarrow} \chi^{2}(p+q-m) .
$$

При альтернативе $H_{1 n}^{L}(\mathbf{d})$

$$
\begin{gathered}
\widehat{L}_{n}^{S}\left(\mathbf{U}, \widehat{\mathbf{c}}_{n 0}\right) \stackrel{d_{\mathbf{c}_{n}}}{\longrightarrow} \chi^{2}\left(p+q-m, \lambda_{S}^{2}(\mathbf{U})\right), \\
\lambda_{S}^{2}(\mathbf{U})=\left(2 g(0) \mathbf{E}\left|\varepsilon_{1}\right|\right)^{2} \mathbf{d}_{\mathbf{U}}^{(2)^{T}} \mathbf{C}_{\mathbf{U}}\left(\mathbf{c}_{0}\right) \mathbf{d}_{\mathbf{U}}^{(2)} .
\end{gathered}
$$

При этом $\lambda_{S}^{2}\left(\mathbf{U}_{1}\right)=\lambda_{S}^{2}\left(\mathbf{U}_{2}\right)$ для любьи $\mathbf{U}_{1}, \mathbf{U}_{2} \in \mathfrak{U}$, m.e. асимптотическое распределение $\widehat{L}_{n}^{S}\left(\mathbf{U}, \widehat{\mathbf{c}}_{n 0}\right)$ одно и тоже для всех $\mathbf{U} \in \mathfrak{U}$.

Доказательство теоремы 4.1 вынесено в раздел 5.

Сравним знаковые тесты (4.6) с классическими и ранговыми. Мы будем цитировать работу [14], в которой рассматривается проверка линейных гипотез в линейной регрессии с погрешностями, удовлетворяющими ARMA соотношению. Это требует весьма громоздких ограничений и кропотливого рассмотрения. При этом линейные гипотезы имеют 
более общий, чем (4.1), вид. Мы перефразируем результаты [14] применительно к нашей ситуации.

Начнем с классических тестов, основанных на векторе $\mathbf{l}_{n, \widehat{\psi}}\left(\widehat{\mathbf{c}}_{n 0}\right)$, в котором $\widehat{\psi}:=\boldsymbol{\psi}\left(\widehat{\mathbf{c}}_{n 0}\right)$, вектор $\mathbf{l}_{n, \psi}(\cdot)$ определен в $(3.8)$. Пусть

$$
\widehat{L}_{n, \widehat{\psi}}\left(\mathbf{U}, \widehat{\mathbf{c}}_{n 0}\right):=\left(\mathbf{l}_{n, \widehat{\psi}}\left(\widehat{\mathbf{c}}_{n 0}\right)\right)^{T} \mathbf{Q}_{\widehat{\psi}}\left(\mathbf{U}, \widehat{\mathbf{c}}_{n 0}\right) \mathbf{l}_{n, \widehat{\psi}}\left(\widehat{\mathbf{c}}_{n 0}\right)
$$

с матрицей $\mathrm{Q}_{\psi}$, равной $\mathrm{Q}_{11}$ из предложения 5.10 в [14].

При гауссовских инновациях асимптотически локально наиболее строгий тест для проверки гипотезы $H^{L}$ против естественной альтернативы (см. [14, предложение 6.1] для уточнений) имеет статистикой квадратическую форму (4.8). В общей ситуации статистика (4.8) имеет при альтернативе $H_{1 n}^{L}(\mathrm{~d})$ из (4.2) асимптотическое распределение $\chi^{2}\left(p-m, \lambda^{2}(\mathbf{U})\right)$. Параметр нецентральности не зависит от $\boldsymbol{\psi}$,

$$
\lambda^{2}(\mathbf{U})=\mathbf{d}_{\mathbf{U}}^{(2)}{ }^{T} \mathbf{C}_{\mathbf{U}}\left(\mathbf{c}_{0}\right) \mathbf{d}_{\mathbf{U}}^{(2)}
$$

Из теоремы 4.1 и соотношения (4.9) следует, что АОЭ знакового теста (4.6) относительно теста (4.8) равна $e_{S}$ из (3.11).

Теперь рассмотрим ранговый тест, основанный на векторе $\mathbf{1}_{n, f, \widehat{\psi}}^{R}\left(\widehat{\mathbf{c}}_{n 0}\right)$ с $\mathbf{l}_{n, f, \psi}^{R}(\cdot)$ из (3.3). Квадратическая ранговая тестовая статистика для $H_{0}^{L}$ имеет вид

$$
\widehat{L}_{n, f, \widehat{\psi}}^{R}\left(\mathbf{U}, \widehat{\mathbf{c}}_{n 0}\right):=\left(\mathbf{l}_{n, f, \widehat{\psi}}^{R}\left(\widehat{\mathbf{c}}_{n 0}\right)\right)^{T} \mathbf{Q}_{\widehat{\psi}}\left(\mathbf{U}, \widehat{\mathbf{c}}_{n 0}\right) \mathbf{l}_{n, f, \widehat{\psi}}^{R}\left(\widehat{\mathbf{c}}_{n 0}\right) .
$$

В силу предложения 5.10 из [14] статистика (4.10) имеет при альтернативе $H_{1 n}^{L}(\mathbf{d})$ асимптотическое распределение $\chi^{2}\left(p-m, \lambda_{R}^{2}(f, \mathbf{U})\right)$ с

$$
\lambda_{R}^{2}(f, \mathbf{U})=I^{2}(f \mid g) \mathbf{d}_{\mathbf{U}}^{(2)^{T}} \mathbf{C}_{\mathbf{U}}\left(\mathbf{c}_{0}\right) \mathbf{d}_{\mathbf{U}}^{(2)}
$$

и $I(f \mid g)$ из (3.6). Из теоремы 4.1 и соотношения (4.11) следует, что АОЭ знакового теста (4.6) относительно рангового (4.10) равна $e_{S, R}(f)$ из (3.7).

При $f=g$ ранговый тест является асимптотически локально наиболее строгим, см. [14, предложение 5.10] для уточнений альтернативы и условий на $g$.

Для знаково-рангового аналога (4.10) справедливы те же асимптотические резупьтаты при дополнительном предположении о симметрии $G$ (см. опять [14, предложение 5.10]).

\section{5. Доказательство теорем.}

Д ок а затель с т в о т е о ремы 3.1. Мы докажем теорему для $p=q=1$, в общем случае доказательство громоздко, но не содержит принципиальных отличий.

Итак, перепишем (1.1) в виде

$$
u_{t}=a u_{t-1}+\varepsilon_{t}+b \varepsilon_{t-1}, \quad t=0, \pm 1, \ldots, \quad|a|<1, \quad|b|<1 .
$$


Для $(5.1) \mathbf{c}=(a, b)^{T}$, и при $H_{1 n}(\mathbf{d}): \mathbf{c}=\mathbf{c}_{n}:=\mathbf{c}_{0}+\mathbf{d} n^{-1 / 2}+o\left(n^{-1 / 2}\right) \mathbf{c}$ $\mathbf{c}_{n}=\left(a_{n}, b_{n}\right)^{T}, a_{n}=a_{0}+d_{1} n^{-1 / 2}+o\left(n^{-1 / 2}\right), b_{n}=b_{0}+d_{2} n^{-1 / 2}+o\left(n^{-1 / 2}\right)$, $\mathbf{d}=\left(d_{1}, d_{2}\right)^{T}$. Остатки

$\varepsilon_{t}(\boldsymbol{\theta})=u_{t}-\theta_{1} u_{t-1}-\theta_{2} \varepsilon_{t-1}(\boldsymbol{\theta}), \quad t=1, \ldots, n ; \quad \varepsilon_{0}(\boldsymbol{\theta})=0, \quad \boldsymbol{\theta}=\left(\theta_{1}, \theta_{2}\right)^{T}$.

Мы опускаем далее индекс $\mathbf{c}_{n}$ у символов вероятности и математического ожидания.

Далее, пусть $\tau=\left(\tau_{1}, \tau_{2}\right)^{T}, \Theta$ фиксировано, $0 \leqslant \Theta<\infty$, и $\Omega_{t}-$ $\sigma$-алгебра, порожденная $\left\{\varepsilon_{i}, i \leqslant t\right\}$.

Лемма 5.1. Пусть справедлива гипотеза $H_{1 n}(\mathbf{d})$. Тодда если выполнены условия (ii), (iv), то

$\varepsilon_{t}\left(\mathbf{c}_{n}+\boldsymbol{\tau} n^{-1 / 2}\right)=\varepsilon_{t}-\tau_{1} n^{-1 / 2} \nu_{t-1}-\tau_{2} n^{-1 / 2} \mu_{t-1}+\sigma_{t}(\boldsymbol{\tau}), \quad t=1, \ldots, n$,

где $\left\{\nu_{t}\right\}$ u $\left\{\mu_{t}\right\}$ представляют собой стаиионарные решения уравнений

$$
\begin{array}{ccc}
\nu_{t}=a_{n} \nu_{t-1}+\varepsilon_{t}, & \mu_{t}=-b_{n} \mu_{t-1}+\varepsilon_{t}, & t=0, \pm 1, \ldots ; \\
n p u \quad n>n_{0} & \sup _{\|\boldsymbol{\tau}\| \leqslant \Theta}\left|\sigma_{t}(\boldsymbol{\tau})\right| \leqslant \widehat{\sigma}_{t}, & t=1, \ldots, n ; \\
& \sup _{n} \sum_{t=1}^{n} \mathbf{E} \widehat{\sigma}_{t}<\infty .
\end{array}
$$

Переменные $\sigma_{t}(\boldsymbol{\tau}), \hat{\sigma}_{t}$ измеримы относительно $\Omega_{t-1}$ для $t=1, \ldots, n$.

Доказа тел л с т во. Пусть $z_{t}:=\varepsilon_{t}\left(\mathbf{c}_{n}+\tau n^{-1 / 2}\right)-\varepsilon_{t}, t=$ $1, \ldots, n, z_{0}:=-\varepsilon_{0}$, а $\delta_{t}:=-\tau_{1} n^{-1 / 2} u_{t-1}-\tau_{2} n^{-1 / 2} \varepsilon_{t-1}$. В силу (5.1) и $(5.2)$

$$
z_{t}=\left(-b_{n}-\tau_{2} n^{-1 / 2}\right) z_{t-1}+\delta_{t}
$$

откуда

$$
\begin{aligned}
z_{t}= & \sum_{j=0}^{t-1}\left(-b_{n}-\tau_{2} n^{-1 / 2}\right)^{j} \delta_{t-j}+\left(-b_{n}-\tau_{2} n^{-1 / 2}\right)^{t} z_{0} \\
= & -\tau_{1} n^{-1 / 2} \sum_{j=0}^{t-1}\left(-b_{n}-\tau_{2} n^{-1 / 2}\right)^{j} u_{t-1-j} \\
& -\tau_{2} n^{-1 / 2} \sum_{j=0}^{t-1}\left(-b_{n}-\tau_{2} n^{-1 / 2}\right)^{j} \varepsilon_{t-1-j}-\left(-b_{n}-\tau_{2} n^{-1 / 2}\right)^{t} \varepsilon_{0} .
\end{aligned}
$$

Последнее выражение может быть переписано в виде

$$
\begin{aligned}
\varepsilon_{t}\left(\mathbf{c}_{n}+\tau n^{-1 / 2}\right)= & \varepsilon_{t}-\tau_{1} n^{-1 / 2} \sum_{j \geqslant 0}\left(-b_{n}\right)^{j} u_{t-1-j} \\
& -\tau_{2} n^{-1 / 2} \sum_{j \geqslant 0}\left(-b_{n}\right)^{j} \varepsilon_{t-1-j}+\sigma_{t}(\tau),
\end{aligned}
$$


где

$$
\begin{aligned}
\sigma_{t}(\tau):= & \tau_{1} n^{-1 / 2} \sum_{j \geqslant t}\left(-b_{n}\right)^{j} u_{t-1-j}-\tau_{1} n^{-1 / 2} \\
& \times \sum_{j=0}^{t-1}\left[\left(-b_{n}-\tau_{2} n^{-1 / 2}\right)^{j}-\left(-b_{n}\right)^{j}\right] u_{t-1-j}+\tau_{2} n^{-1 / 2} \sum_{j \geqslant t}\left(-b_{n}\right)^{j} \varepsilon_{t-1-j} \\
& -\tau_{2} n^{-1 / 2} \sum_{j=0}^{t-1}\left[\left(-b_{n}-\tau_{2} n^{-1 / 2}\right)^{j}-\left(-b_{n}\right)^{j}\right] \varepsilon_{t-1-j} \\
& -\left(-b_{n}-\tau_{2} n^{-1 / 2}\right)^{t} \varepsilon_{0} .
\end{aligned}
$$

Из соотношений (5.7), (5.8) следуют (5.3) и (5.4). Действительно, в модели (5.1)

$$
\nu_{t-1}:=\sum_{j \geqslant 0}\left(-b_{n}\right)^{j} u_{t-1-j}=\sum_{j \geqslant 0} a_{n}^{j} \varepsilon_{t-1-j}
$$

и $\left\{\nu_{t}\right\},\left\{\mu_{t}:=\sum_{j \geqslant 0}\left(-b_{n}\right)^{j} \varepsilon_{t-j}\right\}$ есть стационарные решения уравнений (5.4).

Пусть $0<B<1$ таково, что $\max \left(\left|a_{0}\right|,\left|b_{0}\right|\right)<B$. Пусть

$$
\begin{aligned}
& \xi_{t}:=\sum_{j \geqslant t} B^{j-t}\left(\left|u_{t-1-j}\right|+\left|\varepsilon_{t-1-j}\right|\right), \quad \eta_{t}:=\sum_{j \geqslant 1} j B^{j-1}\left(\left|u_{t-1-j}\right|+\left|\varepsilon_{t-1-j}\right|\right), \\
& \widehat{\sigma}_{t}:=\Theta n^{-1 / 2} B^{t} \xi_{t}+\Theta^{2} n^{-1} \eta_{t}+B^{t}\left|\varepsilon_{0}\right|, \quad 0 \leqslant \Theta<\infty .
\end{aligned}
$$

Определения (5.8) и (5.9) влекут (5.5). В силу условия (ii) $\mathbf{E}\left(\left|\xi_{t}\right|+\left|\eta_{t}\right|+\right.$ $\left.\left|\varepsilon_{t}\right|\right)<\infty$

Отсюда и из (5.9) следует (5.6). Наконец, в силу (5.8) и $(5.9), \sigma_{t}(\boldsymbol{\tau})$ и $\widehat{\sigma}_{t} \Omega_{t-1}$-измеримы. Лемма 5.1 доказана.

При $p=q=1$ имеем $\mathbf{l}_{n}^{S}(\boldsymbol{\theta})=\left(l_{n}^{a}(\boldsymbol{\theta}), l_{n}^{b}(\boldsymbol{\theta})\right)^{T}$ где $l_{n}^{a}(\boldsymbol{\theta})=\sum_{k=1}^{n-1} \theta_{1}^{k-1}(n-$ k) $\Gamma_{k n}(\boldsymbol{\theta}), l_{n}^{b}(\boldsymbol{\theta})=\sum_{k=1}^{n-1} \theta_{2}^{k-1}(n-k) \Gamma_{k n}(\boldsymbol{\theta})$ и

$$
\mathbf{K}(\mathbf{c})=\left[\begin{array}{ll}
\left(1-a^{2}\right)^{-1} & (1+a b)^{-1} \\
(1+a b)^{-1} & \left(1-b^{2}\right)^{-1}
\end{array}\right] .
$$

Для доказательства теоремы 3.1 достаточно показать, что

$$
\begin{aligned}
\sup _{\|\boldsymbol{\tau}\| \leqslant \Theta} \mid & n^{-1 / 2} l_{n}^{a}\left(\mathbf{c}_{n}+\boldsymbol{\tau} n^{-1 / 2}\right)-n^{-1 / 2} \tilde{l}_{n}^{a}\left(a_{0}\right) \\
& +2 g(0) \mathbf{E}\left|\varepsilon_{1}\right|\left[\left(1-a_{0}^{2}\right)^{-1} \tau_{1}+\left(1+a_{0} b_{0}\right)^{-1} \tau_{2}\right] \mid=o_{\mathbf{P}}(1), \\
\sup _{\|\boldsymbol{\tau}\| \leqslant \Theta} \mid & n^{-1 / 2} l_{n}^{b}\left(\mathbf{c}_{n}+\boldsymbol{\tau} n^{-1 / 2}\right)-n^{-1 / 2} \tilde{l}_{n}^{b}\left(b_{0}\right) \\
& +2 g(0) \mathbf{E}\left|\varepsilon_{1}\right|\left[\left(1+a_{0} b_{0}\right)^{-1} \tau_{1}+\left(1-b_{0}^{2}\right)^{-1} \tau_{2}\right] \mid=o_{\mathbf{P}}(1)
\end{aligned}
$$

где

$$
\begin{gathered}
\tilde{l}_{n}^{a}\left(a_{0}\right)=\sum_{k=1}^{n-1} a_{0}^{k-1}(n-k) \widetilde{\Gamma}_{k n}, \quad \widetilde{l}_{n}^{b}\left(b_{0}\right)=\sum_{k=1}^{n-1} b_{0}^{k-1}(n-k) \widetilde{\Gamma}_{k n} \\
\widetilde{\Gamma}_{k n}=(n-k)^{-1} \sum_{t=k+1}^{n} \operatorname{sign}\left(\varepsilon_{t-k} \varepsilon_{t}\right) .
\end{gathered}
$$


Мы докажем (5.10), второе соотношение доказывается аналогично. В силу определений $l_{n}^{a}(\boldsymbol{\theta})$ и $\tilde{l}_{n}^{a}\left(a_{0}\right)$

$$
\begin{aligned}
& n^{-1 / 2} l_{n}^{a}\left(\mathbf{c}_{n}+\boldsymbol{\tau} n^{-1 / 2}\right)-n^{-1 / 2} \tilde{l}_{n}^{a}\left(a_{0}\right) \\
& =n^{-1 / 2} \sum_{k=1}^{n-1}\left(a_{n}+\tau_{1} n^{-1 / 2}\right)^{k-1}(n-k)\left[\Gamma_{k n}\left(\mathbf{c}_{n}+\boldsymbol{\tau} n^{-1 / 2}\right)-\tilde{\Gamma}_{k n}\right] \\
& \quad+n^{-1 / 2} \sum_{k=1}^{n-1}\left[\left(a_{n}+\tau_{1} n^{-1 / 2}\right)^{k-1}-a_{0}^{k-1}\right](n-k) \widetilde{\Gamma}_{k n} .
\end{aligned}
$$

Супремум модуля (5.12) при $\|\boldsymbol{\tau}\| \leqslant \Theta$ есть ор $(1)$, это доказывается аналогично лемме 6.9 .1 в [3]. Рассмотрим (5.11). Пусть

$$
\Delta_{t}(x):= \begin{cases}1, & \varepsilon_{t}<x \\ \frac{1}{2}, & \varepsilon_{t}=x \\ 0, & \varepsilon_{t}>x\end{cases}
$$

Для краткости положим

$$
r_{t-1}=r_{t-1}(\tau):=\tau_{1} n^{-1 / 2} \nu_{t-1}+\tau_{2} n^{-1 / 2} \mu_{t-1}-\sigma_{t}(\tau), \quad t=1, \ldots, n,
$$

где $\nu_{t-1}, \mu_{t-1}$ и $\sigma_{t}(\tau)$ из $(5.4),(5.8)$. В силу (5.3)

$$
\varepsilon_{t}\left(\mathbf{c}_{n}+\boldsymbol{\tau} n^{-1 / 2}\right)=\varepsilon_{t}-r_{t-1}(\boldsymbol{\tau}), \quad t=1, \ldots, n .
$$

В силу (5.14) и определения $\Delta_{t}(x)$ имеем $S_{t}\left(\mathbf{c}_{n}+\boldsymbol{\tau} n^{-1 / 2}\right)=\operatorname{sign}\left(\varepsilon_{t}-\right.$ $\left.r_{t-1}\right)=1-2 \Delta_{t}\left(r_{t-1}\right)$.

Из последнего соотношения и (2.8) получаем, что

$$
n^{-1 / 2}(n-k)\left[\Gamma_{k n}\left(\mathbf{c}_{n}+\boldsymbol{\tau} n^{-1 / 2}\right)-\widetilde{\Gamma}_{k n}\right]=4 x_{k n}(\boldsymbol{\tau})+4 y_{k n}(\boldsymbol{\tau})-2 u_{k n}(\boldsymbol{\tau}),
$$

где

$$
\begin{aligned}
x_{k n}(\boldsymbol{\tau})= & n^{-1 / 2} \sum_{t=k+1}^{n}\left[\Delta_{t-k}\left(r_{t-k-1}\right) \Delta_{t}\left(r_{t-1}\right)-\Delta_{t-k}\left(r_{t-k-1}\right)\right. \\
& \left.\times G\left(r_{t-1}\right)-\Delta_{t-k}(0) \Delta_{t}(0)+\Delta_{t-k}(0) G(0)\right], \\
y_{k n}(\boldsymbol{\tau})= & n^{-1 / 2} \sum_{t=k+1}^{n}\left[\Delta_{t-k}\left(r_{t-k-1}\right) G\left(r_{t-1}\right)-\Delta_{t-k}(0) G(0)\right], \\
u_{k n}(\boldsymbol{\tau})= & n^{-1 / 2} \sum_{t=k+1}^{n}\left[\Delta_{t-k}\left(r_{t-k-1}\right)-\Delta_{t-k}(0)\right] \\
& +n^{-1 / 2} \sum_{t=k+1}^{n}\left[\Delta_{t}\left(r_{t-1}\right)-\Delta_{t}(0)\right] .
\end{aligned}
$$

Используя (5.15), перепишем (5.11) в виде

$$
\sum_{k=1}^{n-1}\left(a_{n}+\tau_{1} n^{-1 / 2}\right)^{k-1}\left[4 x_{k n}(\boldsymbol{\tau})+4 y_{k n}(\boldsymbol{\tau})-2 u_{k n}(\boldsymbol{\tau})\right] .
$$


Лемма 5.2. Пусть условия (ii)-(iv) вьпплнены. Пусть $N_{n} \sim$ $\log _{B}\left(n^{-1}\right)$, где $\left|a_{0}\right|<B<1$. Тогда при $H_{1 n}(\mathbf{d})$

1) $\sup _{k \leqslant N_{n}\|\tau\| \leqslant \Theta} \sup _{\| \sim}\left|x_{k n}(\boldsymbol{\tau})\right|=o_{\mathbf{P}}(1)$;

2) $\sup _{k \leqslant N_{n}} \sup _{\|\boldsymbol{\tau}\| \leqslant \Theta}\left|n^{-1 / 2} \sum_{t=k+1}^{n}\left[\Delta_{t}\left(r_{t-1}\right)-\Delta_{t}(0)\right]\right|=o_{\mathbf{P}}(1)$, $\sup _{k \leqslant N_{n}} \sup _{\|\tau\| \leqslant \Theta}\left|n^{-1 / 2} \sum_{t=k+1}^{n}\left[\Delta_{t-k}\left(r_{t-k-1}\right)-\Delta_{t-k}(0)\right]\right|=o_{\mathbf{P}}(1) ;$

3) $\sup _{k \leqslant N_{n}} \sup _{\|\tau\| \leqslant \Theta}\left|n^{-1 / 2} \sum_{t=k+1}^{n}\left[\Delta_{t-k}\left(r_{t-k-1}\right)-\Delta_{t-k}(0)\right] r_{t-1}\right|=o_{\mathbf{P}}(1)$.

Д о к а з а т е л ь с т в о. Все утверждения доказываются единым методом, поэтому докажем только утверждение пункта 1). Разделим отрезок $\left[-\Theta n^{-1 / 2}, \Theta n^{-1 / 2}\right]$ на $3^{m_{n}}$ частей точками

$$
\eta_{s n}=-\Theta n^{-1 / 2}+2 \Theta n^{-1 / 2} 3^{-m_{n}} s, \quad s=0,1, \ldots, 3^{m_{n}},
$$

и пусть $3^{m_{n}} \sim \log _{B}\left(n^{-1}\right)$. Для $\nu_{t}$ и $\mu_{t}$ из (5.4), положим

$$
\begin{aligned}
& \widehat{\nu}_{t s}:=\nu_{t}\left[1-2 \Theta n^{-1 / 2} 3^{-m_{n}} \eta_{s n}^{-1} I\left(\nu_{t} \leqslant 0\right)\right], \\
& \widetilde{\nu}_{t s}:=\nu_{t}\left[1-2 \Theta n^{-1 / 2} 3^{-m_{n}} \eta_{s n}^{-1} I\left(\nu_{t} \geqslant 0\right)\right],
\end{aligned}
$$

и пусть $\widehat{\mu}_{t s}, \widetilde{\mu}_{t s}$ определены аналогично. Для любых $\tau_{1}, \tau_{2} \in[-\Theta, \Theta]$ выберем $\eta_{l n}$ и $\eta_{f n}$ среди точек $\left\{\eta_{s n}\right\}$ так, что

$$
\begin{aligned}
& 0 \leqslant \eta_{l n}-n^{-1 / 2} \tau_{1} \leqslant 2 \Theta n^{-1 / 2} 3^{-m_{n}}, \\
& 0 \leqslant \eta_{f n}-n^{-1 / 2} \tau_{2} \leqslant 2 \Theta n^{-1 / 2} 3^{-m_{n}} .
\end{aligned}
$$

Из определений $(5.17),(5.18)$ и неравенств (5.19), (5.20) следует, что

$$
\begin{aligned}
\eta_{l n} \widetilde{\nu}_{t-1, l} & \leqslant n^{-1 / 2} \tau_{1} \nu_{t-1} \leqslant \eta_{l n} \widehat{\nu}_{t-1, l}, \\
\eta_{f n} \widetilde{\mu}_{t-1, f} & \leqslant n^{-1 / 2} \tau_{2} \mu_{t-1} \leqslant \eta_{f n} \widehat{\mu}_{t-1, f} .
\end{aligned}
$$

В силу (5.21), (5.22), (5.5) и (5.13)

$$
\widetilde{r}_{t-1, l, f} \leqslant r_{t-1} \leqslant \widehat{r}_{t-1, l, f}
$$

где

$$
\left\{\begin{array}{l}
\widetilde{r}_{t-1, l, f}:=\eta_{l n} \widetilde{\nu}_{t-1, l}+\eta_{f n} \widetilde{\mu}_{t-1, f}-\widehat{\sigma}_{t-1}, \\
\widehat{r}_{t-1, l, f}:=\eta_{l n} \widehat{\nu}_{t-1, l}+\eta_{f n} \widehat{\mu}_{t-1, f}+\widehat{\sigma}_{t-1} .
\end{array}\right.
$$

Пусть $\mathbf{R}_{n}(\boldsymbol{\tau}):=\left(r_{0}(\boldsymbol{\tau}), \ldots, r_{n-1}(\boldsymbol{\tau})\right), \widehat{\mathbf{R}}_{n, l, f}:=\left(\widehat{r}_{0, l, f}, \ldots, \widehat{r}_{n-1, l, f}\right), \widetilde{\mathbf{R}}_{n, l, f}:=$ $\left(\widetilde{r}_{0, l, f}, \ldots, \widetilde{r}_{n-1, l, f}\right)$. Чтобы подчеркнуть зависимость $x_{k n}(\boldsymbol{\tau})$ от компонент $\mathbf{R}_{n}(\boldsymbol{\tau})$, переобозначим $x_{k n}(\boldsymbol{\tau})$ через $x_{k n}\left(\mathbf{R}_{n}(\boldsymbol{\tau})\right)$. Мы так же будем писать $x_{k n}\left(\widehat{\mathbf{R}}_{n, l, f}\right), x_{k n}\left(\widetilde{\mathbf{R}}_{n, l, f}\right)$, если $\widehat{\mathbf{R}}_{n, l, f}, \widetilde{\mathbf{R}}_{n, l, f}$ подставлены вместо $\mathbf{R}_{n}(\boldsymbol{\tau})$ в $x_{k n}\left(\mathbf{R}_{n}(\boldsymbol{\tau})\right)$. Из $(5.23)$ и монотонности $\Delta_{t}(x)$ и $G(x)$ следует, 
чTо

$$
\begin{aligned}
x_{k n}\left(\mathbf{R}_{n}(\boldsymbol{\tau})\right) \leqslant x_{k n}\left(\widehat{\mathbf{R}}_{n, l, f}\right)+n^{-1 / 2} \sum_{t=k+1}^{n}[ & \Delta_{t-k}\left(\widehat{r}_{t-k-1, l, f}\right) G\left(\widehat{r}_{t-1, l, f}\right) \\
& \left.-\Delta_{t-k}\left(\widetilde{r}_{t-k-1, l, f}\right) G\left(\widetilde{r}_{t-1, l, f}\right)\right], \\
x_{k_{n}}\left(\mathbf{R}_{n}(\boldsymbol{\tau})\right) \geqslant x_{k n}\left(\widetilde{\mathbf{R}}_{n, l, f}\right)-n^{-1 / 2} \sum_{t=k+1}^{n}[ & {\left[\Delta_{t-k}\left(\widehat{r}_{t-k-1, l, f}\right) G\left(\widehat{r}_{t-1, l, f}\right)\right.} \\
& \left.-\Delta_{t-k}\left(\widetilde{r}_{t-k-1, l, f}\right) G\left(\widetilde{r}_{t-1, l, f}\right)\right] .
\end{aligned}
$$

Эти неравенства влекут:

$$
\begin{aligned}
\sup _{k \leqslant N_{n}} \sup _{\|\boldsymbol{\tau}\| \leqslant \Theta}\left|x_{k n}\left(\mathbf{R}_{n}(\boldsymbol{\tau})\right)\right| \leqslant \sup _{k \leqslant N_{n}} \sup _{l, f}\left\{\left|x_{k n}\left(\widehat{\mathbf{R}}_{n, l, f}\right)\right|+\left|x_{k n}\left(\widetilde{\mathbf{R}}_{n, l, f}\right)\right|\right\} \\
+\sup _{k \leqslant N_{n}} \sup _{l, f} n^{-1 / 2} \sum_{t=k+1}^{n}\left[\Delta_{t-k}\left(\widehat{r}_{t-k-1, l, f}\right) G\left(\widehat{r}_{t-1, l, f}\right)\right. \\
\left.-\Delta_{t-k}\left(\widetilde{r}_{t-k-1, l, f}\right) G\left(\widetilde{r}_{t-1, l, f}\right)\right] .
\end{aligned}
$$

Рассмотрим первый член, $x_{k n}\left(\widehat{\mathbf{R}}_{n, l, f}\right)$, в $(5.25)$. Положим для краткости

$$
\begin{aligned}
\xi_{t}= & \xi_{t}(n, k, l, f)=\Delta_{t-k}\left(\widehat{r}_{t-k-1, l, f}\right) \Delta_{t}\left(\widehat{r}_{t-1, l, f}\right)-\Delta_{t-k}\left(\widehat{r}_{t-k-1, l, f}\right) G\left(\widehat{r}_{t-1, l, f}\right) \\
& -\Delta_{t-k}(0) \Delta_{t}(0)+\Delta_{t-k}(0) G(0) .
\end{aligned}
$$

Тогда

$$
x_{k n}\left(\widehat{\mathbf{R}}_{n, l, f}\right)=n^{-1 / 2} \sum_{t=k+1}^{n} \xi_{t} .
$$

Пусть $\Omega_{t}$ есть $\sigma$-алгебра, порожденная $\left\{\varepsilon_{i}, i \leqslant t\right\}$. Очевидно, $\widehat{r}_{t-1, l, f}$ и $\widehat{r}_{t-k-1, l, f}$ измеримы относительно $\Omega_{t-1}$. Поскольку $\varepsilon_{t}$ не зависит от $\Omega_{t-1}$, и $\mathbf{E} \Delta_{t}(x)=G(x)$, то $\mathbf{E}\left\{\xi_{t} \mid \Omega_{t-1}\right\}=0$ п.н., т.е. последовательность $\left\{\xi_{t}, \Omega_{t}, t=k+1, k+2, \ldots\right\}$ является мартингал-разностью. Следовательно,

$$
\mathbf{E} \xi_{t}=0, \quad \mathbf{E} \xi_{t} \xi_{s}=0, \quad t \neq s .
$$

Легко проверить, что $\mathbf{E} \xi_{t}^{2} \leqslant 2 \sup _{x} g(x) \mathbf{E}\left(\left|\widehat{r}_{t-k-1, l, f}\right|+\left|\widehat{r}_{t-1, l, f}\right|\right)$. Так как $\min _{s}\left|\eta_{s n}\right|=\Theta n^{-1 / 2}$, то $\max \left(\left|\widehat{\nu}_{t l}\right|,\left|\widehat{\mu}_{t l}\right|\right) \leqslant 3 \max \left(\left|\nu_{t-1}\right|,\left|\mu_{t-1}\right|\right)$. Следовательно, в силу (5.24),

$$
\mathbf{E}\left(\left|\widehat{r}_{t-k-1, l, f}\right|+\left|\widehat{r}_{t-1, l, f}\right|\right) \leqslant c_{1} n^{-1 / 2}+\mathbf{E}\left(\widehat{\sigma}_{t-k-1}+\widehat{\sigma}_{t-1}\right),
$$

где константы $c_{1}, c_{2}, \ldots$ не зависят от $n, k, t, l, f$. Итак, $\mathbf{E} \xi_{t}^{2} \leqslant c_{2}\left(n^{-1 / 2}+\right.$ $\left.\mathbf{E}\left(\widehat{\sigma}_{t-k-1}+\widehat{\sigma}_{t-1}\right)\right)$. Последнее неравенство и $(5.6),(5.27),(5.28)$ влекут

$$
\sup _{k \leqslant N_{n}} \sup _{l, f} \mathbf{E} x_{k n}^{2}\left(\widehat{\mathbf{R}}_{n, l, f}\right)=\sup _{k \leqslant N_{n}} \sup _{l, f} \mathbf{E}\left(n^{-1 / 2} \sum_{t=k+1}^{n} \xi_{t}\right)^{2}=O\left(n^{-1 / 2}\right) .
$$


В силу неравенства Чебышева при любом $\varepsilon>0$

$$
\begin{gathered}
\mathbf{P}\left\{\sup _{k \leqslant N_{n}} \sup _{l, f}\left|x_{k n}\left(\widehat{\mathbf{R}}_{n, l, f}\right)\right|>\varepsilon\right\} \leqslant \sum_{k=1}^{N_{n}} \sum_{l, f=0}^{3^{m_{n}}} \mathbf{P}\left\{\left|x_{k n}\left(\widehat{\mathbf{R}}_{n, l, f}\right)\right|>\varepsilon\right\} \\
\leqslant \varepsilon^{-2} \sum_{k=1}^{N_{n}} \sum_{l, f=0}^{3^{m_{n}}} \mathbf{E} x_{k n}^{2}\left(\widehat{\mathbf{R}}_{n, l, f}\right)=O\left(N_{n} 3^{2 m_{n}} n^{-1 / 2}\right)=o(1)
\end{gathered}
$$

так как $3^{m_{n}}, N_{n} \sim \log _{B}\left(n^{-1}\right)$.

Аналогично показывается, что

$$
\sup _{k \leqslant N_{n}} \sup _{l, f}\left|x_{k n}\left(\tilde{\mathbf{R}}_{n, l, f}\right)\right|=o_{\mathbf{P}}(1) .
$$

Следовательно, (5.25) есть ор(1). Аналогично показывается, что (5.26) есть $o_{\mathbf{P}}(1)$. Лемма 5.2 доказана.

Лемма 5.3. Пусть условия (ii)-(iv) выполненыл. Тогда при $H_{1 n}(\mathbf{d})$

$$
\begin{aligned}
& \sup _{\|\boldsymbol{\tau}\| \leqslant \Theta}\left|\sum_{k=1}^{n-1}\left(a_{n}+\tau_{1} n^{-1 / 2}\right)^{k-1} x_{k n}(\boldsymbol{\tau})\right|=o_{\mathbf{P}}(1) ; \\
& \sup _{\|\boldsymbol{\tau}\| \leqslant \Theta}\left|\sum_{k=1}^{n-1}\left(a_{n}+\tau_{1} n^{-1 / 2}\right)^{k-1} u_{k n}(\boldsymbol{\tau})\right|=o_{\mathbf{P}}(1) .
\end{aligned}
$$

Д о к а з а т е л ь с т в о. Доказательства утверждений пунктов 1) и 2) этой леммы основаны на утверждениях пунктов 1) и 2) леммы 5.2. Поэтому докажем только пункт 1 ). Очевидно, $\left|x_{k n}(\tau)\right| \leqslant 4 n^{1 / 2}$ для $k=1, \ldots, n-1$. Следовательно, для произвольного $\varepsilon>0$ и $B$ такого, что $\left|a_{0}\right|<B<1$, имеем, используя $N_{n} \sim \log _{B}\left(n^{-1}\right)$ :

$$
\begin{aligned}
& \mathbf{P}\left\{\sup _{\|\boldsymbol{\tau}\| \leqslant \Theta}\left|\sum_{k=N_{n}+1}^{n-1}\left(a_{n}+\tau_{1} n^{-1 / 2}\right)^{k-1} x_{k n}(\boldsymbol{\tau})\right|>\varepsilon\right\} \\
& \quad \leqslant \mathbf{P}\left\{4 n^{1 / 2} \sum_{k \geqslant N_{n}+1} B^{k-1}>\varepsilon\right\}=0
\end{aligned}
$$

для всех достаточно больших $n$, поскольку $n^{1 / 2} \sum_{k \geqslant N_{n}+1} B^{k-1}=$ $O\left(n^{1 / 2} B^{N_{n}}\right)=o(1)$. Кроме того, для всех достаточно больших $n$

$$
\sup _{\|\boldsymbol{\tau}\| \leqslant \Theta}\left|\sum_{k=1}^{N_{n}}\left(a_{n}+\tau_{1} n^{-1 / 2}\right)^{k-1} x_{k n}(\boldsymbol{\tau})\right| \leqslant \sup _{k \leqslant N_{n}} \sup _{\|\boldsymbol{\tau}\| \leqslant \Theta}\left|x_{k n}(\boldsymbol{\tau})\right| \sum_{k \geqslant 1} B^{k-1}=o_{\mathbf{P}}(1)
$$

в силу утверждения пункта 1) леммы 5.2. Лемма 5.3 доказана.

Лемма 5.4. Пусть условия (i)-(iv) выполнены. Тогда при гипотезе $H_{1 n}(\mathbf{d})$

$$
\begin{aligned}
\sup _{\|\boldsymbol{\tau}\| \leqslant \Theta} & \mid \sum_{k=1}^{n-1}\left(a_{n}+\tau_{1} n^{-1 / 2}\right)^{k-1} y_{k n}(\boldsymbol{\tau}) \\
& +\frac{1}{2} g(0) \mathbf{E}\left|\varepsilon_{1}\right|\left[\left(1-a_{0}^{2}\right)^{-1} \tau_{1}+\left(1+a_{0} b_{0}\right)^{-1} \tau_{2}\right] \mid=o_{\mathbf{P}}(1) .
\end{aligned}
$$


Д ок аз а т ел в с т в о. Пусть $N_{n} \sim \log _{B} n^{-1}$ с $\left|a_{0}\right|<B<1$. Как при доказательстве леммы 5.3 показывается, что

$$
\sup _{\|\boldsymbol{\tau}\| \leqslant \Theta}\left|\sum_{k=N_{n}+1}^{n-1}\left(a_{n}+\tau_{1} n^{-1 / 2}\right)^{k-1} y_{k n}(\boldsymbol{\tau})\right|=o_{\mathbf{P}}(1) .
$$

В силу определения $y_{k n}(\tau)$,

$$
\begin{aligned}
& \sum_{k=1}^{N_{n}}\left(a_{n}+\tau_{1} n^{-1 / 2}\right)^{k-1} y_{k n}(\tau) \\
& =G(0) \sum_{k=1}^{N_{n}}\left(a_{n}+\tau_{1} n^{-1 / 2}\right)^{k-1} \\
& \quad \times\left\{n^{-1 / 2} \sum_{t=k+1}^{n}\left[\Delta_{t-k}\left(r_{t-k-1}\right)-\Delta_{t-k}(0)\right]\right\} \\
& +\sum_{k=1}^{N_{n}}\left(a_{n}+\tau_{1} n^{-1 / 2}\right)^{k-1} \\
& \times\left\{n^{-1 / 2} \sum_{t=k+1}^{n} \Delta_{t-k}\left(r_{t-k-1}\right)\left[G\left(r_{t-1}\right)-G(0)\right]\right\} .
\end{aligned}
$$

В силу пункта 2) леммы 5.2 слагаемое (5.29) есть $o_{\mathbf{P}}(1)$ равномерно по $\|\boldsymbol{\tau}\| \leqslant \Theta$.

Пусть $\widetilde{r}_{t-1}:=\tau_{1} n^{-1 / 2} \nu_{t-1}+\tau_{2} n^{-1 / 2} \mu_{t-1}$. В силу формулы Тейлора, (5.6) и условий (iii)-(iv) слагаемое (5.30) может быть переписано в виде

$$
\sum_{k=1}^{N_{n}} a_{0}^{k-1}\left\{n^{-1 / 2} \sum_{t=k+1}^{n} \Delta_{t-k}\left(r_{t-k-1}\right) g\left(\theta_{t} \widetilde{r}_{t-1}\right) \widetilde{r}_{t-1}\right\}+o_{\mathbf{P}}(1)
$$

равномерно по $\|\tau\| \leqslant \Theta$ с $\theta_{t} \in(0,1)$. Главный член последнего выражения представим как

$$
\begin{aligned}
& \sum_{k=1}^{N_{n}} a_{0}^{k-1}\left\{n^{-1 / 2} \sum_{t=k+1}^{n} \Delta_{t-k}\left(r_{t-k-1}\right)\left[g\left(\theta_{t} \widetilde{r}_{t-1}\right)-g(0)\right] \widetilde{r}_{t-1}\right\} \\
& \quad+g(0) \sum_{k=1}^{N_{n}} a_{0}^{k-1}\left\{n^{-1 / 2} \sum_{t=k+1}^{n}\left[\Delta_{t-k}\left(r_{t-k-1}\right)-\Delta_{t-k}(0)\right] \widetilde{r}_{t-1}\right\} \\
& \quad+g(0) \sum_{k=1}^{N_{n}} a_{0}^{k-1}\left\{n^{-1 / 2} \sum_{t=k+1}^{n} \Delta_{t-k}(0) \widetilde{r}_{t-1}\right\}
\end{aligned}
$$

Супремум модуля (5.31) по $\|\boldsymbol{\tau}\| \leqslant \Theta$ не больше

$$
\Theta \sum_{k=1}^{N_{n}}\left|a_{0}\right|^{k-1}\left\{n^{-1} \sum_{t=k+1}^{n} \sup _{\|\boldsymbol{\tau}\| \leqslant \Theta}\left|g\left(\widetilde{r}_{t-1}\right)-g(0)\right|\left(\left|\nu_{t-1}\right|+\left|\mu_{t-1}\right|\right)\right\} .
$$


В силу условий (ii)-(iii) математическое ожидание внутренней суммы стремится к нулю. Следовательно, последнее выражение есть ор (1). Значит и (5.31) есть $o_{\mathbf{P}}(1)$ равномерно по $\|\boldsymbol{\tau}\| \leqslant \Theta$. В силу пункта 3 ) леммы 5.2 выражение (5.32) есть ор (1) равномерно по $\|\tau\| \leqslant \Theta$. Теперь рассмотрим (5.33). Перепишем его в виде

$$
\begin{aligned}
& g(0) \tau_{1} \sum_{k=1}^{N_{n}} a_{0}^{k-1}\left\{n^{-1} \sum_{t=k+1}^{n} \Delta_{t-k}(0) \nu_{t-1}\right\} \\
& +g(0) \tau_{2} \sum_{k=1}^{N_{n}} a_{0}^{k-1}\left\{n^{-1} \sum_{t=k+1}^{n} \Delta_{t-k}(0) \mu_{t-1}\right\} .
\end{aligned}
$$

Рассмотрим (5.34). Для фиксированного $1 \leqslant l \leqslant N_{n}$ имеем

$$
\sum_{k=1}^{N_{n}} a_{0}^{k-1}\left\{n^{-1} \sum_{t=k+1}^{n} \Delta_{t-k}(0) \nu_{t-1}\right\}=X_{l n}+Y_{l n}
$$

где

$$
\begin{aligned}
& X_{l n}:=\sum_{k=1}^{l} a_{0}^{k-1}\left\{n^{-1} \sum_{t=k+1}^{n} \Delta_{t-k}(0) \nu_{t-1}\right\}, \\
& Y_{l n}:=\sum_{k=l+1}^{N_{n}} a_{0}^{k-1}\left\{n^{-1} \sum_{t=k+1}^{n} \Delta_{t-k}(0) \nu_{t-1}\right\} .
\end{aligned}
$$

При условиях (ii) и (iv) для фиксированного $k$

$$
n^{-1} \sum_{t=k+1}^{n} \Delta_{t-k}(0) \nu_{t-1}=-\frac{1}{2} \mathbf{E}\left|\varepsilon_{1}\right| a_{0}^{k-1}+o_{\mathbf{P}}(1) .
$$

Следовательно, для фиксированного $l$ и $n \rightarrow \infty, X_{l n}$ слабо сходится к $-\frac{1}{2} \mathbf{E}\left|\varepsilon_{1}\right| \sum_{k=1}^{l} a_{0}^{2(k-1)}$. Последнее выражение сходится к $-\frac{1}{2} \mathbf{E}\left|\varepsilon_{1}\right|\left(1-a_{0}^{2}\right)^{-1}$ при $l \rightarrow \infty$. Поскольку $\sup _{n} \mathbf{E}\left|Y_{l n}\right| \leqslant c\left|a_{0}\right|^{l} \rightarrow 0, l \rightarrow \infty$, то, в силу теоремы 7.7 .1 из [1],

$$
\sum_{k=1}^{N_{n}} a_{0}^{k-1}\left\{n^{-1} \sum_{t=k+1}^{n} \Delta_{t-k}(0) \nu_{t-1}\right\}=-\frac{1}{2} \mathbf{E}\left|\varepsilon_{1}\right|\left(1-a_{0}^{2}\right)^{-1}+o_{\mathbf{P}}(1) .
$$

Следовательно, (5.34) есть $-\frac{1}{2} g(0) \mathbf{E}\left|\varepsilon_{1}\right|\left(1-a_{0}^{2}\right)^{-1} \tau_{1}+o \mathbf{P}(1)$ равномерно по $\left|\tau_{1}\right| \leqslant \Theta$. Похожими рассуждениями показывается, что (5.35) есть $-\frac{1}{2} g(0) \mathbf{E} \mid \varepsilon_{1}\left(1+a_{0} b_{0}\right)^{-1} \tau_{2}+o \mathbf{P}(1)$ равномерно по $\left|\tau_{2}\right| \leqslant \Theta$. Итак, (5.33) есть

$$
-\frac{1}{2} g(0) \mathbf{E}\left|\varepsilon_{1}\right|\left[\left(1-a_{0}^{2}\right)^{-1} \tau_{1}+\left(1+a_{0} b_{0}\right)^{-1} \tau_{2}\right]+o_{\mathbf{P}}(1)
$$

равномерно по $\|\boldsymbol{\tau}\| \leqslant \Theta$. Доказательство леммы 5.4 завершено.

Из леммы 5.3 и 5.4 следует, что (5.16) есть

$$
-2 g(0) \mathbf{E}\left|\varepsilon_{1}\right|\left[\left(1-a_{0}^{2}\right)^{-1} \tau_{1}+\left(1+a_{0} b_{0}\right)^{-1} \tau_{2}\right]+\varepsilon_{n}(\tau),
$$

где $\sup _{\|\boldsymbol{\tau}\| \leqslant \Theta}\left|\varepsilon_{n}(\boldsymbol{\tau})\right|=o_{\mathbf{P}}(1)$. Теорема 3.1 доказана. 
Док аз а тель с тво т е о рем ы.1. По предположению, $n^{1 / 2}\left(\widehat{\mathbf{c}}_{n}-\mathbf{c}_{n}\right)=O_{\mathbf{P}}(1)$ при $H_{1 n}^{L}(\mathbf{d})$, следовательно, и $n^{1 / 2}\left(\widehat{\mathbf{c}}_{n 0}-\mathbf{c}_{n}\right)=O_{\mathbf{P}}(1)$. В силу определения (4.4) и свойств матрицы $\mathrm{U}$

$$
\mathbf{W}_{\mathbf{U}}\left(\mathbf{c}_{0}\right) \mathbf{W}_{\mathbf{U}}^{T}\left(\mathbf{c}_{0}\right)=\mathbf{U K}^{-1}\left(\mathbf{c}_{0}\right) \mathbf{U}
$$

Используя (5.36), следствие 3.1 и состоятельность $\widehat{\mathrm{W}}_{n, P}$ получаем:

$$
\begin{aligned}
n^{-1 / 2} & \widehat{\mathbf{W}}_{n, \mathbf{U}}^{T} \mathbf{U} \mathbf{l}_{n}^{S}\left(\widehat{\mathbf{c}}_{n 0}\right) \\
= & n^{-1 / 2} \widehat{\mathbf{W}}_{n, \mathbf{U}}^{T} \mathbf{U} \widetilde{\mathbf{l}}_{n}^{S}\left(\mathbf{c}_{0}\right)-2 g(0) \mathbf{E}\left|\varepsilon_{1}\right| \widehat{\mathbf{W}}_{n, \mathbf{U}}^{T} \mathbf{U K}\left(\mathbf{c}_{0}\right) n^{1 / 2}\left(\widehat{\mathbf{c}}_{n 0}-\mathbf{c}_{n}\right)+o_{\mathbf{P}}(1) \\
= & n^{-1 / 2} \mathbf{W}_{\mathbf{U}}^{T}\left(\mathbf{c}_{0}\right) \mathbf{U i}{ }_{n}^{S}\left(\mathbf{c}_{0}\right)-2 g(0) \mathbf{E}\left|\varepsilon_{1}\right| \mathbf{W}_{\mathbf{U}}^{T}\left(\mathbf{c}_{0}\right) \mathbf{U K}\left(\mathbf{c}_{0}\right) \\
& \times n^{1 / 2}\left(\widehat{\mathbf{c}}_{n 0}-\mathbf{c}_{n}\right)+o_{\mathbf{P}}(1)=n^{-1 / 2} \mathbf{W}_{\mathbf{U}}^{T}\left(\mathbf{c}_{0}\right) \mathbf{U} \widetilde{\mathbf{l}}_{n}^{S}\left(\mathbf{c}_{0}\right)-2 g(0) \mathbf{E}\left|\varepsilon_{1}\right| \\
& \times \mathbf{W}_{\mathbf{U}}^{-1}\left(\mathbf{c}_{0}\right)\left(n^{1 / 2}\left(\widehat{\mathbf{c}}_{n}^{(1)}-\mathbf{c}_{n}^{(1)}\right)^{T},-\mathbf{d}_{\mathbf{U}}^{(2) T}\right)^{T}+o_{\mathbf{P}}(1) .
\end{aligned}
$$

Поскольку

$$
\mathbf{W}_{\mathbf{U}}^{-1}\left(\mathbf{c}_{0}\right)=\left(\begin{array}{cc}
\mathbf{J}_{\mathbf{U}}^{1 / 2}\left(\mathbf{c}_{0}\right) & \mathbf{J}_{\mathbf{U}}^{-1 / 2}\left(\mathbf{c}_{0}\right) \mathbf{B}_{\mathbf{U}}^{T}\left(\mathbf{c}_{0}\right) \\
0 & \mathbf{C}_{\mathbf{U}}^{1 / 2}\left(\mathbf{c}_{0}\right)
\end{array}\right)
$$

получаем:

$$
\begin{aligned}
\pi \circ n^{-1 / 2} \widehat{\mathbf{W}}_{n, \mathbf{U}}^{T} \mathbf{U l}_{n}^{S}\left(\widehat{\mathbf{c}}_{n 0}\right)= & \pi \circ n^{-1 / 2} \mathbf{W}_{\mathbf{U}}^{T}\left(\mathbf{c}_{0}\right) \widetilde{\mathbf{U l}}_{n}^{S}\left(\mathbf{c}_{0}\right) \\
& +2 g(0) \mathbf{E}\left|\varepsilon_{1}\right| \mathbf{C}_{\mathbf{U}}^{1 / 2}\left(\mathbf{c}_{0}\right) \mathbf{d}_{\mathbf{U}}^{(2)}+o_{\mathbf{P}}(1) .
\end{aligned}
$$

Последнее соотношение влечет сходимость

$$
\widehat{L}_{n}^{S}\left(\mathbf{U}, \widehat{\mathbf{c}}_{n 0}\right) \stackrel{d_{\mathbf{c}_{n}}}{\longrightarrow} \chi^{2}\left(p+q-m, \lambda_{S}^{2}(\mathbf{U})\right) .
$$

Покажем, что параметр нецентральности $\lambda_{S}^{2}(\mathbf{U})$ один и тот же при всех U. Выделим среди всех матриц $\mathfrak{U}$ одну, обозначим ее $\mathbf{U}_{0}$. Тогда $\mathbf{U}=\mathbf{U}_{12} \mathbf{U}_{0}$, где $\mathbf{U}_{12}=\left(\begin{array}{cc}\mathbf{U}_{1} & \mathbf{0} \\ \mathbf{0} & \mathbf{U}_{2}\end{array}\right)$, матрицы $\mathbf{U}_{i}, i=1,2$, получаются перестановкой строк матриц $\mathbf{I}_{m}$ и $\mathbf{I}_{p+q-m}$. Очевидно, $\mathbf{U}_{i}-$ симметрические ортогональные матрицы, $\mathbf{U}_{i}=\mathbf{U}_{i}^{-1}$. Пользуясь свойствами блочных матриц и представлением (4.3) получаем:

$$
\mathbf{U K}(\mathbf{c}) \mathbf{U}=\mathbf{U}_{12} \mathbf{U}_{0} \mathbf{K}(\mathbf{c}) \mathbf{U}_{0} \mathbf{U}_{12}=\left(\begin{array}{cc}
\mathbf{U}_{1} \mathbf{J}_{\mathbf{U}_{0}}(\mathbf{c}) \mathbf{U}_{1} & \mathbf{U}_{1} \mathbf{B}_{\mathbf{U}_{\mathrm{o}}}^{T}(\mathbf{c}) \mathbf{U}_{2} \\
\mathbf{U}_{2} \mathbf{B}_{\mathbf{U}_{0}}(\mathbf{c}) \mathbf{U}_{1} & \mathbf{U}_{2} \mathbf{M}_{\mathbf{U}_{0}}(\mathbf{c}) \mathbf{U}_{2}
\end{array}\right) .
$$

Отсюда, в силу определения (4.5) и свойств $\mathbf{U}_{i}, \mathbf{C}_{\mathbf{U}}(\mathbf{c})=\mathbf{U}_{2} \mathbf{C}_{\mathbf{U}_{0}}(\mathbf{c}) \mathbf{U}_{2}$. Следовательно,

$$
\begin{aligned}
\lambda_{S}^{2}(\mathbf{U}) & =\left(2 g(0) \mathbf{E}\left|\varepsilon_{1}\right|\right)^{2}\left(\mathbf{U}_{2} \mathbf{d}_{\mathbf{U}}^{(2)}\right)^{T} \mathbf{C}_{\mathbf{U}_{0}}\left(\mathbf{c}_{0}\right)\left(\mathbf{U}_{2} \mathbf{d}_{\mathbf{U}}^{(2)}\right) \\
& =\left(2 g(0) \mathbf{E}\left|\varepsilon_{1}\right|\right)^{2}\left(\mathbf{U}_{2}^{-1} \mathbf{d}_{\mathbf{U}}^{(2)}\right)^{T} \mathbf{C}_{\mathbf{U}_{0}}\left(\mathbf{c}_{0}\right)\left(\mathbf{U}_{2}^{-1} \mathbf{d}_{\mathbf{U}}^{(2)}\right) \\
& =\left(2 g(0) \mathbf{E}\left|\varepsilon_{1}\right|\right)^{2} \mathbf{d}_{\mathbf{U}_{0}}^{(2)^{T}} \mathbf{C}_{\mathbf{U}_{0}}\left(\mathbf{c}_{0}\right) \mathbf{d}_{\mathbf{U}_{0}}^{(2)}=\lambda_{S}^{2}\left(\mathbf{U}_{0}\right) .
\end{aligned}
$$

Теорема 4.1 доказана. 


\section{СПИСОК ЛИТЕРАТУРЫ}

1. Anderson T.W. The Statistical Analysis of Time Series. New York: Wiley, 1971, $704 \mathrm{p}$.

2. Bloomfield P., Steiger W.L. Least Absolute Deviation. Theory, Applications and Algorithms. Boston: Birkhäuser, 1983, 349 p.

3. Boldin M. V., Simonova G. I., Tyurin Yu. N. Sign-based Methods in Linear Statistical Models. Providence, RI: Amer. Math. Society, 1997, 234 p.

4. Brockwell P. J., Davis R. A. Time Series: Theory and Methods. New York: SpringerVerlag, 1987, $519 \mathrm{p}$.

5. Davis R. A., and Resnick S. I. Limit theory for the sample covariance and correlation functions of moving averages. - Ann. Statist., 1986, v. 14, № 2, p. 533-558.

6. Dufour J. M. Rank tests for serial dependence. - J. Time Ser. Anal., 1981, v. 2, № 3, p. $117-128$.

7. Dufour J. M., Hallin M., Mizera I. Generalized runs tests for heteroscedastic time series. - J. Nonparametric Statist., 1998, v. 9, № 1, p. 39-86.

8. Hájek J., Šilák Z. Theory of Rank Tests. New York: Academic Press, 1967, 297 p.

9. Hallin M., Ingenbleek J.-F., Puri M. L. Linear serial rank tests for randomness against ARMA alternatives. - Ann. Statist., 1985, v. 13, № 3, p. 1156-1181.

10. Hallin J., Ingenbleek J.-F., Puri M. L. Linear and quadratic rank tests for randomness against serial dependence. - J. Time Ser. Anal., 1987, v. 8, № 4, p. 409-424.

11. Hallin M., Puri M. L. Optimal rank-based procedures for time series analysis: testing an ARMA model against other ARMA models. - Ann. Statist., 1988, v. 16, № 1, p. 402-432.

12. Hallin M., Puri M.L. Time series analysis via rank order theory: signed-rank tests for ARMA models. - J. Multivariate Analysis, 1991, v. 39, № 1, p. 1-29.

13. Hallin M., Puri M. L. Rank tests for time-series analysis: a survey. New Directions in Time Series Analysis, Part I. Ed. by D. Brillinger, E. Parzen, and M. Rosenblatt. New York: Springer, 1992, p. 111-153.

14. Hallin M., Puri M.L. Aligned rank tests for linear models with autocorrelated error terms. - J. Multivariate Anal., 1994, v. 50, № 2, p. 175-237.

15. Hannan E. J., Kanter M. Autoregressive processes with infinite variance. -- J. Appl. Probab., 1977, v. 14, № 2, p. 411-415.

16. Kanter M., Steiger W.L. Regression and autoregression with infinite variance. Adv. Probab., 1974, v. 6, p. 768-783.

17. Koul H.L. Weighted Empiricals and Linear Models. Hayward: Institute of Mathematical Statistics, IMS, 1992, 264 p.

18. Kreiss J. P. On adaptive estimation in stationary ARMA processes. - Ann. Statist., 1987 , v. 15 , № 1 , p. 112-133.

19. Kreiss J. P. Testing linear hypotheses in autoregressions. - Ann. Statist., 1990, v. 18, № 3, p. 1470-1482.

20. Le Cam L. Asymptotic Methods in Statistical Decision Theory. New York: SpringerVerlag, 1986, $742 \mathrm{p}$.

21. Mikosch T., Gadrich T., Klüppelberg C., Adler R. J. Parametric estimation for ARMA models with infinite variance innovations. - Ann. Statist., 1995, v. 23, № 1, p. 305326.

22. Pollard D. Asymptotics for least absolute deviation regression estimators. Econometric Theory, 1991, v. 7, № 2, p. 186-199.

23. Strasser H. Mathematical Theory of Statistics. Berlin: de Gruyter, 1985, 492 p.

24. Yohai V.J., Maronna R.A. Asymptotic behavior of least-squares estimates for autoregressive processes with infinite variances. -- Ann. Statist., 1977, v. 5, p. 554560 . 